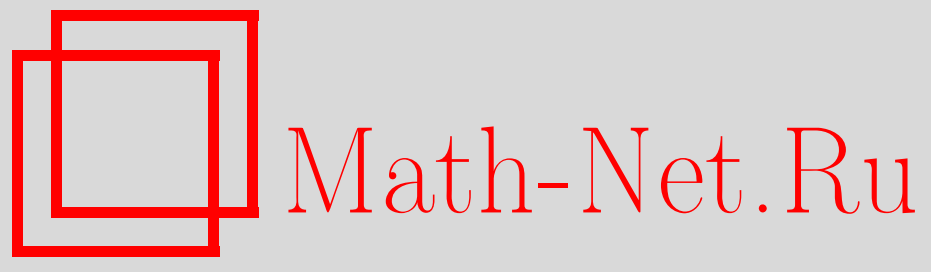

Е. А. Неганова, В. И. Трофимов, Симметрические расширения графов, Изв. РАН. Сер. матем., 2014, том 78, выпуск 4, 175-206

DOI: https://doi.org/10.4213/im8054

Использование Общероссийского математического портала Math-Net.Ru подразумевает, что вы прочитали и согласны с пользовательским соглашением http://www.mathnet.ru/rus/agreement

Параметры загрузки:

IP: 34.239 .49 .27

26 апреля 2023 г., 04:35:42

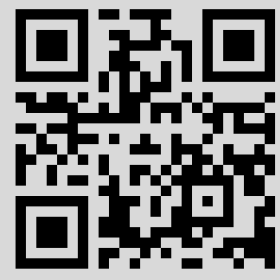


УДК $512.54+519.17$

\author{
Е. А. Неганова, В. И. Трофимов
}

\title{
Симметрические расширения графов
}

Исследуются симметрические расширения графов, причем особое внимание уделяется симметрическим и $\operatorname{Aut}_{0}\left(\Lambda^{d}\right)$-симметрическим расширениям $d$-мерных решеток $\Lambda^{d}$ посредством конечных графов. Рассматриваемые вопросы представляют интерес для теории групп и теории графов, а также могут представлять интерес для кристаллографии и некоторых разделов физики. Доказывается существование связного локально конечного графа, имеющего бесконечное число симметрических расширений посредством фиксированного конечного графа. С другой стороны, в ряде представляющих интерес случаев доказывается конечность числа симметрических и $\operatorname{Aut}_{0}\left(\Lambda^{d}\right)$-симметрических расширений $d$-мерной решетки $\Lambda^{d}$ посредством конечного графа. Кроме того, для каждого целого положительного числа $d$ строятся все $\operatorname{Aut}_{0}\left(\Lambda^{d}\right)$-симметрические расширения $d$-мерной решетки $\Lambda^{d}$ посредством графов с двумя вершинами.

Библиография: 15 наименований.

Ключевые слова: симметрическое расширение графов, граф Кэли группы, $d$-мерная решетка, автоморфизмы графа.

DOI: $10.4213 / \operatorname{im} 8054$

\section{§1. Введение}

Пусть $\Gamma$ и $\Delta$ - графы (под графом в настоящей работе понимается неориентированный граф без петель и без кратных ребер). Следуя [1], назовем связный граф $\widetilde{\Gamma}$ симметрическим расширением графа $\Gamma$ посредством графа $\Delta$, если существуют такая вершинно-транзитивная группа $\widetilde{G}$ автоморфизмов графа $\widetilde{\Gamma}$ и такая система импримитивности $\sigma$ группы $\widetilde{G}$ на множестве $V(\widetilde{\Gamma})$ вершин графа $\widetilde{\Gamma}$, что факторграф $\widetilde{\Gamma} / \sigma$ изоморфен графу $\Gamma$ и блоки системы $\sigma$ порождают в $\widetilde{\Gamma}$ подграфы, изоморфные графу $\Delta$. Ясно, что симметрическое расширение графа $\Gamma$ посредством графа $\Delta$ существует лишь для $\Gamma$ и $\Delta$, допускающих транзитивные на вершинах группы автоморфизмов, причем граф $Г$ должен быть связным. (В случае, если граф Г имеет более одной вершины, эти условия являются также достаточными для существования симметрического расширения $\Gamma$ посредством $\Delta$. Действительно, в этом случае в качестве симметри-

Работа выполнена при финансовой поддержке РФФИ (грант № 10-01-00349-а), Программы отделения математических наук РАН (проект 12-Т-1-1003) и Программы совместных исследований УрО РАН с СО РАН (проект 12-С-1-1018); кроме того, работа первого автора поддержана грантом УрО РАН для молодых ученых (проект А3, 2012 г.), а работа второго автора выполнена в рамках проекта, подпадающего под Соглашение между Министерством образования и науки РФ и Уральским федеральным университетом (№ 02.А03.21.0006 от 27.08.2013). 
ческого расширения $Г$ посредством $\Delta$ может быть взято, например, лексикографическое произведение графов $Г$ и $\Delta$; см. [2].) Если в приведенном выше определении симметрического расширения графа $\Gamma$ посредством графа $\Delta$ отказаться от условия связности графа $\widetilde{\Gamma}$, то получим определение обобщенного симметрического расширения графа Г посредством графа $\Delta$. При этом если $\varphi-$ изоморфизм графа $\widetilde{\Gamma} / \sigma$ на граф $\Gamma$, то будем говорить, что $\widetilde{\Gamma}-$ обобщенное симметрическое расширение графа $\Gamma$ посредством графа $\Delta$, реализуемое $\widetilde{G}, \sigma, \varphi$.

Симметрические расширения графов представляют интерес в силу целого ряда причин (см. ниже). При этом часто бывает важно, чтобы (мы используем обозначения из приводимого выше определения симметрического расширения $\Gamma$ посредством $\Delta$ ) при изоморфизме $\widetilde{\Gamma} / \sigma$ на $\Gamma$ индуцируемая $\widetilde{G}$ группа $\widetilde{G}^{\sigma}$ автоморфизмов графа $\widetilde{\Gamma} / \sigma$ переходила в некоторую заданную группу автоморфизмов $G$ графа $\Gamma$. В связи с этим, следуя [1], для графов $\Gamma, \Delta$ и вершинно-транзитивной группы автоморфизмов $G$ графа $\Gamma$ связный граф $\widetilde{\Gamma}$ назовем $G$-симметрическим расширением графа $\Gamma$ посредством графа $\Delta$, если граф $\widetilde{\Gamma}$ является симметрическим расширением графа $\Gamma$ посредством графа $\Delta$, реализуемым такими $\widetilde{G}, \sigma, \varphi$, что $\varphi \widetilde{G}^{\sigma} \varphi^{-1}=G$. Если в этом определении отказаться от условия связности графа $\widetilde{\Gamma}$, то получим определение обобщенного $G$-симметрического расширения графа $\Gamma$ посредством графа $\Delta$ (реализуемого $\widetilde{G}, \sigma, \varphi)$.

Укажем некоторые направления исследований, где (обобщенные) симметрические расширения графов возникают естественным образом, а их изучение может представлять интерес (ср. [1]).

1. Понятие симметрического расширения одного графа посредством другого графа аналогично хорошо известному понятию расширения одной группы посредством другой группы. Связь между расширениями групп и симметрическими расширениями графов можно формализовать следующим образом. Если $\widetilde{G}$ - группа с системой порождающих $\widetilde{M}$ такой, что $1 \notin \widetilde{M}=\widetilde{M}^{-1}$, и $N-$ нормальная подгруппа группы $\widetilde{G}$, то для группы $G=\widetilde{G} / N$ и ее естественного действия на своем графе Кэли $\Gamma_{G, M}$, построенном по системе порождающих $M=\{g N: g \in \widetilde{M} \backslash N\}$, граф Кэли $\Gamma_{\widetilde{G}} \widetilde{M}$ группы $\widetilde{G}$, построенный по системе порождающих $\widetilde{M}$, является $G$-симметрическим расширением $\Gamma_{G, M}$ посредством графа Кэли $\Gamma_{N, \widetilde{M} \cap N}$ группы $N$, построенного по множеству $\widetilde{M} \cap N$. (Определение графа Кэли группы, построенного по ее подмножеству, не содержащему 1 и замкнутому относительно операции взятия обратного элемента, см. в §2.) В связи с этим изучение симметрических расширений графов представляет интерес для теории групп.

2. Для вершинно-симметрических (т. е. допускающих транзитивные на вершинах группы автоморфизмов) графов $Г$ и $\Delta$ ряд известных конструкций теории графов, таких, например, как декартово, прямое, лексикографическое и некоторые другие произведения (см. [2]), приводят к симметрическим расширениям $Г$ посредством $\Delta$. Далее, если $\Gamma$ - связный граф, допускающий вершинно-транзитивную группу автоморфизмов $G$, и если $\psi: V(\widetilde{\Gamma}) \rightarrow V(\Gamma)-$ накрывающее отображение связного графа $\widetilde{\Gamma}$ на граф $\Gamma$ такое, что соответствующая ему накрывающая группа $\widetilde{G}:=\{g \in \operatorname{Aut}(\widetilde{\Gamma}): \psi g \in G \psi\}$ группы $G$ 
вершинно-транзитивна, то множество слоев $\sigma:=\left\{\psi^{-1}(x): x \in V(\Gamma)\right\}$ есть система импримитивности $\widetilde{G}$ (на $V(\widetilde{\Gamma}))$ и $\psi$ индуцирует изоморфизм $\varphi$ графа $\widetilde{\Gamma} / \sigma$ на $\Gamma$ такой, что $\varphi \widetilde{G}^{\sigma} \varphi^{-1}-$ вершинно-транзитивная подгруппа группы $G$. Следовательно, $\widetilde{\Gamma}$ является $\left(\varphi \widetilde{G}^{\sigma} \varphi^{-1}\right)$-симметрическим расширением $\Gamma$ посредством $\Delta$, где $\Delta$ - подграф графа $\widetilde{\Gamma}$, порожденный некоторым слоем из $\sigma$.

3. Ранее для ряда важных классов связных бесконечных локально конечных вершинно-симметрических графов были получены описания, имеющие по существу следующий вид: графы из рассматриваемого класса являются в точности $G$-симметрическими расширениями некоторых известных графов $Г$ посредством конечных графов, где $G$ - некоторые заданные группы автоморфизмов графов Г. Указанного вида описания были получены, например, для графов с полиномиальным ростом (см. [3]), для графов с рекуррентным случайным блужданием (см. [4]) и для графов с вершинно-транзитивной группой ограниченных автоморфизмов (см. [5]). Изучение таких $G$-симметрических расширений графов Г посредством конечных графов является, таким образом, более детальным исследованием этих классов. Определенный интерес представляет, следовательно, изучение симметрических расширений бесконечных локально конечных графов посредством конечных графов.

4. Если $\widetilde{\Gamma}$ - симметрическое расширение графа $\Gamma$ посредством графа $\Delta$, то граф $\widetilde{\Gamma}$ можно интерпретировать "кристаллографически" как граф $\Gamma$, вершины которого наделены внутренней структурой вида $\Delta$ (такие наделенные внутренней структурой вершины графа Г являются своего рода "молекулами" вида $\Delta$ ), причем эти внутренние структуры вершин графа Г согласуются со структурой $\Gamma$ так, что вся получающаяся в результате конструкция $\widetilde{\Gamma}$ симметрична (т. е. граф $\widetilde{\Gamma}$ допускает вершинно-транзитивную группу автоморфизмов, отображающих "молекулы" на "молекулы"). В связи с этим для кристаллографии частиц с внутренней структурой интерес представляют симметрические расширения $d$-мерных решеток $\Lambda^{d}$ (определение см. в $\S 2$ ) посредством конечных графов. Определенный интерес представляет следующий вопрос: верно ли, что для любого целого положительного числа $d$ и любого конечного графа $\Delta$ существует лишь конечное число симметрических расширений $d$-мерной решетки $\Lambda^{d}$ посредством графа $\Delta$ ?

5. В некоторых физических теориях (см., например, [6]) пространство-время наряду с $d$ "обычными размерностями" имеет несколько "компактифицированных размерностей". В качестве трансляционно-однородных дискретных аппроксимаций такого пространства-времени могут выступать $\operatorname{Aut}_{0}\left(\Lambda^{d}\right)$-симметрические расширения $d$-мерной решетки $\Lambda^{d}$ посредством конечных графов, где $\operatorname{Aut}_{0}\left(\Lambda^{d}\right)$ - группа всех сдвигов решетки $\Lambda^{d}$. (Под трансляционной однородностью здесь понимается возможность перемещения любой вершины в любую другую вершину автоморфизмом, индуцирующим сдвиг $\Lambda^{d}$, т. е. трансляцию на "обычных размерностях" пространства-времени.) В связи с этим представляют интерес $\operatorname{Aut}_{0}\left(\Lambda^{d}\right)$-симметрические расширения $d$-мерных решеток $\Lambda^{d}$ посредством конечных графов.

В настоящей работе нас интересуют симметрические расширения бесконечных локально конечных графов $Г$ и, в особенности, $\operatorname{Aut}_{0}\left(\Lambda^{d}\right)$-симметрические 
расширения $d$-мерных решеток $\Lambda^{d}$ посредством конечных графов $\Delta$. При этом нас интересует, прежде всего, вопрос о конечности их числа для заданных $\Gamma$ (или $d$ в случае решеток) и $\Delta$. В $\S 3$ настоящей работы доказывается, что существует весьма широкий класс локально конечных графов, каждый из которых имеет бесконечное число симметрических расширений посредством фиксированного конечного графа (существование таких графов ранее было анонсировано в [1]). Это является первым основным результатом статьи. Остается, однако, открытым следующий вопрос (см. [1, вопрос 3]): верно ли, что для любого целого положительного числа $d$ и любого конечного графа $\Delta$ существует лишь конечное число $\operatorname{Aut}_{0}\left(\Lambda^{d}\right)$-симметрических расширений $d$-мерной решетки $\Lambda^{d}$ посредством графа $\Delta$ ? (Отметим, что открытым является и аналогичный вопрос для симметрических расширений $d$-мерных решеток.) $\mathrm{B} \S 4$ настоящей работы дается положительный ответ на этот вопрос в случае, когда число вершин графа $\Delta$ есть произвольное простое число (а также в некоторых других случаях; подробнее содержание $\$ 4$, посвященного исследованию вопросов о конечности числа симметрических и $\operatorname{Aut}_{0}\left(\Lambda^{d}\right)$-симметрических расширений $d$-мерной решетки $\Lambda^{d}$ посредством конечного графа, мы обсудим в заключительной части введения). Это является вторым основным результатом статьи. Далее, в силу указанных выше причин наряду с вопросом о конечности числа $\operatorname{Aut}_{0}\left(\Lambda^{d}\right)$-симметрических расширений $d$-мерной решетки $\Lambda^{d}$ посредством конечного графа интерес представляет описание всех (обобщенных) $\operatorname{Aut}_{0}\left(\Lambda^{d}\right)$-симметрических расширений $d$-мерной решетки $\Lambda^{d}$ посредством конечного графа $\Delta$ для фиксированных (как правило, небольших) $d$ и $\Delta$. В $\S 5$ получено такое описание для произвольного целого положительного числа $d$ и произвольного графа $\Delta$ с двумя вершинами. Это является третьим основным результатом статьи. Отметим, что в [7]-[9] получено описание $\operatorname{Aut}_{0}\left(\Lambda^{d}\right)$-симметрических расширений $d$-мерной решетки $\Lambda^{d}$ посредством графа $\Delta$ для всех $d \leqslant 2$ и $\Delta$ с тремя и четырьмя вершинами.

Как было указано выше, симметрические расширения графов естественным образом возникают при исследовании расширений групп, а также в некоторых направлениях исследований в теории графов. Однако развитые в связи с этим методы их исследования применимы, как правило, лишь к так называемым накрывающим симметрическим расширениям графов. (Это же справедливо и для соответствующим образом модифицированных методов теории накрывающих пространств.) Под накрывающим симметрическим расширением графа Г (соответственно, накрывающим G-симметрическим расширением графа Г, где $G$ - вершинно-транзитивная группа автоморфизмов графа $\Gamma)$ посредством графа $\Delta$ понимается симметрическое (соответственно, $G$-симметрическое) расширение $\widetilde{\Gamma}$ графа $\Gamma$ посредством графа $\Delta$, для которого $\sigma$ (см. определение симметрического (соответственно, $G$-симметрического) расширения $\Gamma$ посредством $\Delta$ в начале статьи) может быть выбрана со следующим дополнительным свойством: для произвольной вершины $x$ графа $\widetilde{\Gamma}$ каждый блок системы $\sigma$, смежный в графе $\widetilde{\Gamma} / \sigma$ с $x^{\sigma}$, содержит в точности одну смежную с $x$ вершину. (Когда говорится, что накрывающее симметрическое (соответственно, $G$-симметрическое) расширение графа $\Gamma$ посредством графа $\Delta$ реализуется $\widetilde{G}$, 
$\sigma, \varphi$, то подразумевается, что $\sigma$ обладает указанным дополнительным свойством.) Отметим, что фактически в 33 строится весьма широкий класс локально конечных графов, каждый из которых имеет бесконечное число именно накрывающих симметрических расширений посредством фиксированного конечного графа. В $\S 4$ доказывается, что, в отличие от случая произвольного локально конечного вершинно-симметрического графа, $d$-мерная решетка для каждого целого положительного числа $d$ имеет лишь конечное число накрывающих симметрических расширений посредством любого фиксированного конечного графа (см. следствие 4.3).

В целом, $\$ 4$ работы посвящен исследованию вопросов о конечности числа симметрических (п. 4.1) и $\operatorname{Aut}_{0}\left(\Lambda^{d}\right)$-симметрических (п. 4.2) расширений $d$-мерной решетки $\Lambda^{d}$ посредством конечного графа. Наш подход к исследованию этих вопросов основывается на проверке выполнения для симметрических и $\operatorname{Aut}_{0}\left(\Lambda^{d}\right)$-симметрических расширений $d$-мерной решетки $\Lambda^{d}$ посредством конечного графа следующего условия $\left[n_{1}, \ldots, n_{d}\right]$-периодичности. Пусть $\Gamma$ - обобщенное симметрическое расширение решетки $\Lambda^{d}$ посредством конечного графа, реализуемое некоторыми $G, \sigma, \varphi$. Скажем, что $(\Gamma, \sigma, \varphi)$ удовлетворяет условию $\left[n_{1}, \ldots, n_{d}\right]$-периодичности, где $n_{1}, \ldots, n_{d}$ - целые положительные числа, если существуют такие автоморфизмы $g_{1}, \ldots, g_{d}$ графа $\Gamma$, сохраняющие разбиение $\sigma$, что $\left[g_{i}, g_{j}\right]=1$ для всех $1 \leqslant i<j \leqslant d$ и $\varphi g_{i}^{\sigma} \varphi^{-1}=t_{i}^{n_{i}}$ для всех $1 \leqslant i \leqslant d$. (Это определение не зависит от выбора вершинно-транзитивной группы $G$ автоморфизмов графа $\Gamma$, для которой $\sigma$ является системой импримитивности.)

Несложно показать (см. теорему 4.1), что для произвольных целых чисел $d \geqslant 1, n_{1}, \ldots, n_{d} \geqslant 1$ и произвольного конечного графа $\Delta$ существует лишь конечное число графов $\Gamma$, являющихся обобщенными симметрическими расширениями решетки $\Lambda^{d}$ посредством графа $\Delta$, реализуемыми такими $G, \sigma, \varphi$, что $(\Gamma, \sigma, \varphi)$ удовлетворяет условию $\left[n_{1}, \ldots, n_{d}\right]$-периодичности. Таким образом, для доказательства конечности числа обобщенных симметрических расширений $d$-мерной решетки $\Lambda^{d}$ посредством конечного графа $\Delta$ достаточно показать, что каждое из них может быть реализовано такими $G, \sigma, \varphi$, что $(\Gamma, \sigma, \varphi)$ удовлетворяет условию $\left[n_{1}, \ldots, n_{d}\right]$-периодичности для некоторых фиксированных $n_{1}, \ldots, n_{d}$. Именно этим способом доказываются в $\S 4$ результаты о конечности числа симметрических и $\operatorname{Aut}_{0}\left(\Lambda^{d}\right)$-симметрических расширений $d$-мерной решетки $\Lambda^{d}$ посредством конечного графа. В связи с изложенным уместно сформулировать следующие вопросы (cp. [1, вопросы 4, 5]), представляющие также самостоятельный интерес. Пусть Г - симметрическое (или $\operatorname{Aut}_{0}\left(\Lambda^{d}\right)$-симметрическое) расширение $d$-мерной решетки $\Lambda^{d}$ посредством произвольного конечного графа $\Delta$, реализуемое некоторыми $G, \sigma, \varphi$. Верно ли, что $(\Gamma, \sigma, \varphi)$ удовлетворяет условию $\left[n_{1}, \ldots, n_{d}\right]$-периодичности для некоторых целых положительных чисел $n_{1}, \ldots, n_{d}$ ? Могут ли при этом числа $n_{1}, \ldots, n_{d}$ быть выбраны зависящими только от $d$ и $\Delta ?$ Как было отмечено выше, из положительного ответа на последний вопрос для заданных $d$ и $\Delta$ следует (согласно теореме 4.1) конечность числа симметрических (соответственно, $\operatorname{Aut}_{0}\left(\Lambda^{d}\right)$-симметрических) расширений $d$-мерной решетки $\Lambda^{d}$ посредством $\Delta$. Отметим также, что для произвольного конечного графа $\Delta$ при $d=1$ 
положительный ответ на последний вопрос очевиден, а при $d=2$ положительный ответ на первый вопрос следует из [10, предложение 2].

Как будет видно из дальнейшего, в ряде интересных случаев симметрические расширения решетки $\Lambda^{d}, d$ - целое положительное число, посредством конечного графа $\Delta$ удовлетворяют условию $\left[n_{1}, \ldots, n_{d}\right]$-периодичности, где $n_{i}$, $1 \leqslant i \leqslant d$, ограничены сверху величинами, зависимость которых от графа $\Delta$ сводится к зависимости от числа его вершин. В связи с этим нам будет удобно ввести следующие общие определения. Для произвольного целого положительного числа $q$ связный граф $\widetilde{\Gamma}$ называется симметрическим q-расширением графа Г (соответственно, $G$-симметрическим q-расширением графа $\Gamma$, где $G$ вершинно-транзитивная группа автоморфизмов графа $\Gamma)$, если $\widetilde{\Gamma}$ - симметрическое (соответственно, $G$-симметрическое) расширение графа Г посредством некоторого графа с $q$ вершинами. Если в этом определении отказаться от условия связности графа $\widetilde{\Gamma}$, то получим определение обобщенного симметрического (соответственно, G-симметрического) q-расширения графа Г.

Многие представляющие интерес симметрические расширения решетки $\Lambda^{d}$, $d$ - целое положительное число, посредством конечного графа допускают реализацию такими $G, \sigma, \varphi$, что группа $G$ имеет конечный стабилизатор вершины. В теореме 4.2 настоящей работы доказывается, что если $Г$ - обобщенное симметрическое $q$-расширение решетки $\Lambda^{d}$ для некоторых целых положительных чисел $d$ и $q$, реализуемое $G, \sigma, \varphi$, причем группа $G$ имеет конечный стабилизатор вершины, то $(\Gamma, \sigma, \varphi)$ удовлетворяет условию $\left[n_{1}, \ldots, n_{d}\right]$-периодичности, где числа $n_{i}, 1 \leqslant i \leqslant d$, ограничены сверху величинами, зависящими (явно указываемым образом) лишь от $d, q$ и порядка стабилизатора вершины в группе $G$. С учетом теоремы 4.1 отсюда следует, что для произвольных целых положительных чисел $d, q$ и $m$ существует лишь конечное число обобщенных симметрических $q$-расширений решетки $\Lambda^{d}$, которые могут быть реализованы такими $G, \sigma, \varphi$, что порядок стабилизатора вершины в группе $G$ не превосходит $m$ (см. следствие 4.2). В связи с этим было бы интересно исследовать для произвольных заданных $d$ и $q$ вопрос о существовании одного и того же числа $m$ с этим свойством для всех $q$-симметрических расширений решетки $\Lambda^{d}$ (предварительно исследовав, возможно, вопрос о реализуемости обобщенных симметрических $q$-расширений решетки $\Lambda^{d}$ такими $G, \sigma, \varphi$, что $G$ имеет конечный стабилизатор вершины).

В п. 4.2 с использованием теоремы 4.2 доказывается, что если $\Gamma-\operatorname{Aut}_{0}\left(\Lambda^{d}\right)$ симметрическое $q$-расширение решетки $\Lambda^{d}$ для некоторого целого положительного числа $d$ и некоторого целого положительного простого числа $q$, реализуемое $G, \sigma, \varphi$, то $(\Gamma, \sigma, \varphi)$ удовлетворяет условию $\left[n_{1}, \ldots, n_{d}\right]$-периодичности, где числа $n_{i}, 1 \leqslant i \leqslant d$, ограничены сверху величинами, зависящими (явно указываемым образом) лишь от $d$ и $q$ (см. следствие 4.5). С учетом теоремы 4.1 это влечет отмеченный выше результат о конечности числа $\operatorname{Aut}_{0}\left(\Lambda^{d}\right)$-симметрических $q$-расширений решетки $\Lambda^{d}$ для произвольного целого положительного числа $d$ и произвольного целого положительного простого числа $q$ (см. следствие 4.5$)$. (В п. 4.2 аналогичные результаты доказываются в более общем случае, когда вместо простоты числа $q$ предполагается лишь квазипримитивность действия 
на блоке системы импримитивности $\sigma$ стабилизатора этого блока в группе $G$; см. теорему 4.3 и следствие 4.4.)

\section{§ 2. Определения и вспомогательные результаты}

Используемая в работе терминология, в основном, стандартна.

Если Г - граф (напомним, что в настоящей работе рассматриваются только неориентированные графы без петель и без кратных ребер), то $V(\Gamma)$ - множество его вершин и $E(\Gamma)$ - множество его ребер (ребра рассматриваются как двухэлементные подмножества множества $V(\Gamma))$. Граф Г называется локально конечным, если валентности всех его вершин конечны.

Для связного графа $Г$ через $d_{\Gamma}$ обозначается обычная метрика на $V(\Gamma)$, $d_{\Gamma}(x, y)$ для $x, y \in V(\Gamma)$ есть длина кратчайшего пути между $x$ и $y$ в графе $\Gamma$. Для графа $\Gamma$ и целого положительного числа $n$ через $\Gamma^{n}$ обозначается граф такой, что $V\left(\Gamma^{n}\right)=V(\Gamma)$ и $\{x, y\} \in E\left(\Gamma^{n}\right)$ тогда и только тогда, когда $x \neq y$ и между $x$ и $y$ в графе $\Gamma$ найдется путь длины, не превосходящей $n$.

Для графа $\Gamma$ и $X \subseteq V(\Gamma)$ через $\langle X\rangle_{\Gamma}$ обозначается подграф графа $\Gamma$, порожденный $X$. Для графа $\Gamma$ и разбиения $\sigma$ множества $V(\Gamma)$ через $\Gamma / \sigma$ обозначается факторграф графа Г по разбиению $\sigma$. Для $x \in V(\Gamma)$ через $x^{\sigma}$ обозначается при этом подмножество из $\sigma$, содержащее $x$ (таким образом, $x^{\sigma} \in V(\Gamma / \sigma)$ ).

Мы рассматриваем изоморфизм графа $\Gamma_{1}$ на граф $\Gamma_{2}$ как биекцию $V\left(\Gamma_{1}\right)$ на $V\left(\Gamma_{2}\right)$, отображающую смежные вершины в смежные, а несмежные вершины в несмежные. Соответственно, мы рассматриваем автоморфизм графа Г как подстановку на $V(\Gamma)$, отображающую смежные вершины в смежные, а несмежные вершины в несмежные. Группа всех автоморфизмов графа Г обозначается через $\operatorname{Aut}(\Gamma)$.

В настоящей работе, говоря о конечности или бесконечности числа графов с определенным свойством, мы, как обычно, понимаем число таких графов с точностью до изоморфизма.

Если $g$ - автоморфизм графа Г и $X-g$-инвариантное подмножество множества $V(\Gamma)$, то $g^{X}$ - подстановка на $X$, индуцируемая $g$. Соответственно, если $G$ - группа автоморфизмов графа $\Gamma$ и $X-G$-инвариантное подмножество множества $V(\Gamma)$, то $G^{X}$ - группа подстановок на $X$, индуцируемая $G$. Если $g$ - автоморфизм графа $\Gamma$, сохраняющий разбиение $\sigma$ множества $V(\Gamma)$, то $g^{\sigma}-$ автоморфизм графа $\Gamma / \sigma$, индуцируемый $g$. Соответственно, если $G$ - группа автоморфизмов графа $\Gamma$, сохраняющая разбиение $\sigma$ множества $V(\Gamma)$, то $G^{\sigma}-$ группа автоморфизмов графа $\Gamma / \sigma$, индуцируемая $G$.

Если $G$ - группа автоморфизмов графа $\Gamma, x \in V(\Gamma)$ и $X \subseteq V(\Gamma)$, то $G_{x}=\{g \in G: g(x)=x\}-$ стабилизатор $x$ в $G, G_{X}=\left\{g \in G: g\left(x^{\prime}\right)=\right.$ $x^{\prime}$ для всех $\left.x^{\prime} \in X\right\}$ - поэлементный стабилизатор $X$ в $G, G_{\{X\}}=\{g \in G$ : $g(X)=X\}-$ глобальный стабилизатор $X$ в $G$.

Как обычно, если $G$ - вершинно-транзитивная группа автоморфизмов графа $\Gamma$, то разбиение $\sigma$ множества $V(\Gamma)$ такое, что $g(X) \in \sigma$ для всех $g \in G$, и $X \in \sigma$ называется системой импримитивности группы $G$. Элементы систем импримитивности группы $G$ называются блоками импримитивности группы $G$. 
Напомним еще ряд стандартных определений, используемых в настоящей работе.

Если $G$ - группа и $M=M^{-1}$ - подмножество группы $G$, не содержащее 1 , то графом Кэли группы $G$, построенным по $M$, называется граф $\Gamma_{G, M}$ с множеством вершин $V\left(\Gamma_{G, M}\right)=G$ и множеством ребер $E\left(\Gamma_{G, M}\right)=\{\{g, g a\}: g \in G$, $a \in M\}$. Действие $G$ левыми сдвигами на $V\left(\Gamma_{G, M}\right)$ есть действие автоморфизмами графа $\Gamma_{G, M}$ (и называется естественным действием группы $G$ на графе $\left.\Gamma_{G, M}\right)$. Граф $\Gamma_{G, M}$ связен тогда и только тогда, когда $M-$ система порождающих группы $G$.

Пусть $d$ - целое положительное число. Под $d$-мерной решеткой $\Lambda^{d}$ мы понимаем граф, вершинами которого являются все упорядоченные наборы $\left(a_{1}, \ldots, a_{d}\right)$ из $d$ целых чисел, и две вершины $\left(a_{1}^{\prime}, \ldots, a_{d}^{\prime}\right)$ и $\left(a_{1}^{\prime \prime}, \ldots, a_{d}^{\prime \prime}\right)$ смежны тогда и только тогда, когда выполнено условие

$$
\left|a_{1}^{\prime}-a_{1}^{\prime \prime}\right|+\cdots+\left|a_{d}^{\prime}-a_{d}^{\prime \prime}\right|=1
$$

Для $d$-мерной решетки $\Lambda^{d}$ и $1 \leqslant j \leqslant d$ мы полагаем $\operatorname{Pr}_{j}: V\left(\Lambda^{d}\right) \rightarrow \mathbb{Z}$, $\operatorname{Pr}_{j}\left(\left(a_{1}, a_{2}, \ldots, a_{d}\right)\right)=a_{j}$.

Сдвигом решетки $\Lambda^{d}$ называется ее автоморфизм, который переводит произвольную вершину $\left(a_{1}, \ldots, a_{d}\right)$ в вершину $\left(a_{1}+k_{1}, \ldots, a_{d}+k_{d}\right)$ для некоторых фиксированных целых чисел $k_{1}, \ldots, k_{d}$. Для каждого $1 \leqslant i \leqslant d$ через $t_{i}$ обозначается сдвиг решетки $\Lambda^{d}$, для которого $k_{i}=1$ и $k_{j}=0$ при всех $j \in\{1, \ldots, d\} \backslash\{i\}$. Через $\operatorname{Aut}_{0}\left(\Lambda^{d}\right)$ обозначается подгруппа группы автоморфизмов решетки $\Lambda^{d}$, состоящая из всех ее сдвигов. Ясно, что $\operatorname{Aut}_{0}\left(\Lambda^{d}\right)=$ $\left\langle t_{1}, \ldots, t_{d}\right\rangle \cong \mathbb{Z}^{d}$ и $\Lambda^{d}$ изоморфна графу Кэли группы $\operatorname{Aut}_{0}\left(\Lambda^{d}\right)$, построенному по системе порождающих $\left\{t_{1}^{ \pm 1}, \ldots, t_{d}^{ \pm 1}\right\}$. В соответствии с $\S 1$ для произвольного целого положительного числа $q$ связный граф Г называется симметрическим (соответственно, $\operatorname{Aut}_{0}\left(\Lambda^{d}\right)$-симметрическим) q-расширением решетки $\Lambda^{d}$, если существуют такая вершинно-транзитивная группа $G$ автоморфизмов графа Г и такая система импримитивности $\sigma$ группы $G$ на $V(\Gamma)$ с блоками порядка $q$, что найдется изоморфизм $\varphi$ графа $\Gamma / \sigma$ на решетку $\Lambda^{d}$ (соответственно, найдется изоморфизм $\varphi$ графа $\Gamma / \sigma$ на решетку $\Lambda^{d}$, для которого $\left.\varphi G^{\sigma} \varphi^{-1}=\operatorname{Aut}_{0}\left(\Lambda^{d}\right)\right)$. При этом будем говорить, что $\Gamma-$ симметрическое (соответственно, $\operatorname{Aut}_{0}\left(\Lambda^{d}\right)$-симметрическое) $q$-расширение решетки $\Lambda^{d}$, реализуемое $G, \sigma, \varphi$.

Напомним (см. [5]), что для произвольного связного графа $Г$ через $\operatorname{Aut}_{0}(\Gamma)$ обозначается группа всех его ограниченных автоморфизмов, т. е. таких автоморфизмов $g$, что расстояния в графе Г между $x$ и $g(x)$, где $x$ пробегает множество всех вершин графа Г, ограничены в совокупности. (Введенное выше обозначение $\operatorname{Aut}_{0}\left(\Lambda^{d}\right)$ согласуется с этим определением.) Из [5, следствие 2, (i)] легко следует, что если $\Gamma$ - связный локально конечный граф, то множество $T\left(\operatorname{Aut}_{0}(\Gamma)\right)$ всех ограниченных автоморфизмов конечного порядка графа $\Gamma$ является (нормальной) подгруппой группы Aut(Г). Следующий результат, вытекающий из [5, следствие 1], используется далее при доказательстве теоремы 3.1. 
(Он также используется в [1] при доказательстве теоремы 2, приводимой ниже как предложение 2.2 , непосредственными следствиями которой являются предложения 4.1, 4.2 настоящей работы.)

ПРЕДЛОЖЕНИЕ 2.1. Пусть Г - бесконечный связный локалъно конечный граф такой, что $\operatorname{Aut}_{0}(\Gamma)$ - вериинно-транзитивная группа его автоморфизмов. Тогда множество $T\left(\mathrm{Aut}_{0}(\Gamma)\right)$ элементов конечного порядка групnъ $\operatorname{Aut}_{0}(\Gamma)$ является (нормальной) подгруппой с конечными орбитами группы Aut(Г), причем для системы импримитивности $\sigma$ группы $\operatorname{Aut}_{0}(\Gamma)$ на $V(\Gamma)$, состоящей из $T\left(\operatorname{Aut}_{0}(\Gamma)\right)$-орбит, имеем $\operatorname{Aut}_{0}(\Gamma)^{\sigma} \cong \mathbb{Z}^{d}$, где $d$ - целое положительное число.

Следующее предложение потребуется нам в $₫ 4$.

ПрЕДЛОЖЕНИЕ 2.2 [1, теорема 2]. Пусть Г - связный локалъно конечный граф, $G$ - вериинно-транзитивная группа автоморфизмов графа $\Gamma$ и $\sigma$ - система импримитивности группы $G$ на $V(\Gamma)$ с конечными блоками. Предположим, что каждый неединичный элемент группы $G^{\sigma}$ имеет бесконечный порядок и действует без неподвижных точек на $\sigma$. Тогда блоки $\sigma$ являются $T\left(\operatorname{Aut}_{0}(\Gamma)\right)$-орбитами на $V(\Gamma)$.

Как отмечено в [1] (см. также предложение 4.1 ниже), из предложения 2.2 вытекает, что если $\Gamma-\operatorname{Aut}_{0}\left(\Lambda^{d}\right)$-симметрическое расширение решетки $\Lambda^{d}, d-$ целое положительное число, посредством конечного графа, реализуемое $G$, $\sigma, \varphi$, то блоки $\sigma$ однозначно определены (они являются $T\left(\operatorname{Aut}_{0}(\Gamma)\right.$ )-орбитами на $V(\Gamma))$ и $Г$ является $\operatorname{Aut}_{0}\left(\Lambda^{d}\right)$-симметрическим расширением решетки $\Lambda^{d}$, реализуемым $\operatorname{Aut}_{0}(\Gamma), \sigma, \varphi$.

ЗАмЕчАниЕ 2.1. Если $\widetilde{\Gamma}-$ симметрическое расширение графа $Г$ посредством графа $\Delta$, реализуемое $\widetilde{G}, \sigma, \varphi$, то в ряде случаев (в связи, например, с пп. 1,4 из $\S 1)$ вместо $\widetilde{\Gamma}$ предпочтительно рассматривать пару $(\widetilde{\Gamma}, \sigma)$. При этом пара $(\widetilde{\Gamma}, \sigma)$ считается эквивалентной паре $\left(\widetilde{\Gamma^{\prime}}, \sigma^{\prime}\right)$ (где $\widetilde{\Gamma^{\prime}}-$ также симметрическое расширение графа $\Gamma$ посредством графа $\Delta$, реализуемое некоторыми $\left.\widetilde{G^{\prime}}, \sigma^{\prime}, \varphi^{\prime}\right)$, если существует изоморфизм графа $\widetilde{\Gamma}$ на граф $\widetilde{\Gamma^{\prime}}$, который переводит разбиение $\sigma$ в разбиение $\sigma^{\prime}$. Более того, в случае, когда для заданной вершинно-транзитивной группы автоморфизмов $G$ графа $\Gamma$ граф $\widetilde{\Gamma}$ есть $G$-симметрическое расширение графа $\Gamma$ посредством графа $\Delta$, реализуемое $\widetilde{G}$, $\sigma, \varphi$, вместо пары $(\widetilde{\Gamma}, \sigma)$ предпочтительно рассматривать тройку $(\widetilde{\Gamma}, \sigma, \widetilde{G})$. При этом тройка $(\widetilde{\Gamma}, \sigma, \widetilde{G})$ считается эквивалентной тройке $\left(\widetilde{\Gamma^{\prime}}, \sigma^{\prime}, \widetilde{G^{\prime}}\right)$ (где $\widetilde{\Gamma^{\prime}}$ - также симметрическое расширение графа $\Gamma$ посредством графа $\Delta$, реализуемое некоторыми $\left.\widetilde{G^{\prime}}, \sigma^{\prime}, \varphi^{\prime}\right)$, если существует изоморфизм $\psi$ графа $\widetilde{\Gamma}$ на граф $\widetilde{\Gamma^{\prime}}$, который переводит разбиение $\sigma$ в разбиение $\sigma^{\prime}$ и для которого $\left(\psi G \psi^{-1}\right)^{\sigma^{\prime}}=\left(G^{\prime}\right)^{\sigma^{\prime}}$. Однако для целей настоящей работы такое рассмотрение пар и троек нам не потребуется. Отметим лишь, что согласно утверждению, непосредственно предшествующему настоящему замечанию, в случае, когда $\Gamma=\Lambda^{d}-d$-мерная решетка, где $d \geqslant 1, \Delta$ - конечный граф и $G=\operatorname{Aut}_{0}\left(\Lambda^{d}\right)$, произвольные тройки $(\widetilde{\Gamma}, \sigma, \widetilde{G}),\left(\widetilde{\Gamma^{\prime}}, \sigma^{\prime}, \widetilde{G^{\prime}}\right)$ эквивалентны. 


\section{§ 3. Существование графов с бесконечным числом расширений посредством конечного графа}

В настоящем параграфе доказывается существование связного локально конечного графа $\Gamma$ и конечного графа $\Delta$ таких, что имеется бесконечное число симметрических расширений $\Gamma$ посредством $\Delta$ (этот результат был ранее анонсирован в [1]). Более того, нами строится весьма широкий класс графов $\Gamma$, имеющих для любого целого числа $q>1$ бесконечное число накрывающих симметрических расширений посредством одного и того же конечного графа $\Delta$ с $q$ вершинами (см. замечание 3.1 далее).

Tеорема 3.1. Существует группа $G$ с конечной системой порождающих $M=M^{-1}, 1 \notin M$, такая, что для любого иелого числа $q>1$ графб Кэли $\Gamma_{G, M}$ группы $G$, построенный по системе порождающих $M$, имеет бесконечное число накрывающих $G$-симметрических, относительно естественного действия $G$ на $\Gamma_{G, M}, q$-расширений.

ДокАЗАтельство. Пусть $A$ - группа с системой порождающих $\left\{a_{1}^{ \pm 1}, \ldots\right.$ $\left.\ldots, a_{d}^{ \pm 1}\right\}$, где $d$ - целое положительное число, и $B$ - центральная подгруппа группы $A$ такие, что выполнены следующие условия:

1) $a_{k} a_{l}^{-1} \notin B$ для всех $1 \leqslant k \leqslant d, 1 \leqslant l \leqslant d, k \neq l$, и $a_{k} a_{l} \notin B$ для всех $1 \leqslant k \leqslant d, 1 \leqslant l \leqslant d$

2) $B$ - свободная абелева группа счетного ранга;

3) порядок любой конечной подгруппы группы $A / B$ не превосходит некоторого натурального числа $r$.

(Примеры таких $A,\left\{a_{1}^{ \pm 1}, \ldots, a_{d}^{ \pm 1}\right\}$ и $B$ будут приведены в конце параграфа.)

Положим $G=A / B$, и пусть $\varphi: A \rightarrow A / B=G$ - естественный гомоморфизм группы $A$ на группу $G$. Пусть, кроме того, $M:=\left\{\varphi\left(a_{1}^{ \pm 1}\right), \ldots, \varphi\left(a_{d}^{ \pm 1}\right)\right\}$. Покажем, что для каждого целого числа $q>1$ граф Кэли $\Gamma_{G, M}$ группы $G$, построенный по системе порождающих $M$, имеет бесконечное число накрывающих $G$-симметрических, относительно естественного действия $G$ на $\Gamma_{G, M}$, $q$-расширений.

Пусть $q$ - произвольное целое число, большее единицы. С учетом 2) имеем $Z(A) \geqslant B=\left\langle b_{1}\right\rangle \times\left\langle b_{2}\right\rangle \times \cdots$, где $\left\langle b_{i}\right\rangle \cong \mathbb{Z}$ для всех целых положительных чисел $i$. Положим $B_{1}=\left\langle b_{1}^{q}\right\rangle \times\left\langle b_{2}\right\rangle \times \cdots$ и $B_{i}=\left\langle b_{1}\right\rangle \times \cdots \times\left\langle b_{i-1}\right\rangle \times\left\langle b_{i}^{q}\right\rangle \times\left\langle b_{i+1}\right\rangle \times \cdots$ для всех целых чисел $i>1$.

Пусть $i$ - произвольное целое положительное число. Положим $G_{i}=A / B_{i}$, и пусть $\varphi_{i}$ - естественный гомоморфизм $A \rightarrow A / B_{i}=G_{i}$. Тогда множество $M_{i}:=\left\{\varphi_{i}\left(a_{1}^{ \pm 1}\right), \ldots, \varphi_{i}\left(a_{d}^{ \pm 1}\right)\right\}$ является системой порождающих группы $G_{i}$, причем с учетом 1) имеем $M_{i}=M_{i}^{-1}, 1 \notin M_{i}$. Далее, $K_{i}:=B / B_{i}$ - циклическая подгруппа порядка $q$ группы $Z\left(G_{i}\right)$ и группа $G_{i} / K_{i}=\left(A / B_{i}\right) /\left(B / B_{i}\right)$ естественным образом изоморфна группе $G$. Отождествим группу $G_{i}$ с индуцируемой ей при естественном действии группой автоморфизмов графа Кэли $\Gamma_{G_{i}, M_{i}}$. Тогда для системы импримитивности $\sigma_{i}$ группы $G_{i}$ на $V\left(\Gamma_{G_{i}, M_{i}}\right)$, состоящей из $K_{i}$-орбит, справедливы следующие утверждения:

а) блоки $\sigma_{i}$ имеют порядок $q$; 
b) для произвольной вершины $x$ графа $\Gamma_{G_{i}, M_{i}}$ каждый блок системы $\sigma_{i}$, смежный в графе $\Gamma_{G_{i}, M_{i}} / \sigma_{i}$ с $x^{\sigma_{i}}$, содержит в точности одну смежную с $x$ вершину;

c) естественный изоморфизм группы $G_{i} / K_{i}$ на $G$ индуцирует изоморфизм $\psi_{i}$ графа $\Gamma_{G_{i}, M_{i}} / \sigma_{i}$ на граф $\Gamma_{G, M}$, для которого $\psi_{i} G_{i}^{\sigma_{i}} \psi_{i}^{-1}=G$.

Таким образом, для каждого целого положительного числа $i$ граф $\Gamma_{G_{i}, M_{i}}$ является накрывающим $G$-симметрическим $q$-расширением графа $\Gamma_{G, M}$, peализуемым $G_{i}, \sigma_{i}, \psi_{i}$. Покажем, что среди графов $\Gamma_{G_{i}, M_{i}}$ при целом положительном $i$ имеется бесконечное множество попарно неизоморфных графов. Предположим противное. Тогда среди графов $\Gamma_{G_{i}, M_{i}}$ существует бесконечное множество $\left\{\Gamma_{G_{i_{j}}, M_{i_{j}}}: j\right.$ - целое неотрицательное число $\}$ попарно изоморфных графов. Положим $\Xi_{j}=\Gamma_{G_{i_{j}}, M_{i_{j}}}, \tau_{j}=\sigma_{i_{j}}$ для каждого целого неотрицательного числа $j$. Пусть, кроме того, $\chi_{j}$ - изоморфизм $\Xi_{j}$ на $\Xi_{0}, j \geqslant 1$. Выберем произвольную вершину $x_{0}$ графа $\Xi_{0}$ и положим $x_{j}=\chi_{j}^{-1}\left(x_{0}\right) \in V\left(\Xi_{j}\right)$ для каждого целого положительного числа $j$.

Для каждого целого неотрицательного числа $j$ обозначим через $l_{j}$ длину кратчайшего пути графа $\Xi_{j}$, начинающегося в $x_{j}$ и заканчивающегося в $x_{j}^{\tau_{j}} \backslash\left\{x_{j}\right\}$. Тогда $l_{j}$ - длина кратчайшей записи неединичного элемента из $K_{i_{j}}$ через $M_{i_{j}}$. Другими словами, $l_{j}$ - длина кратчайшего слова над $\left\{a_{1}^{ \pm 1}, \ldots, a_{d}^{ \pm 1}\right\}$ с тем свойством, что представляемый им элемент группы $A$ содержится в $B \backslash B_{i_{j}}$. Отсюда следует, что для любого целого положительного числа $c$ найдется такое целое положительное число $n_{c}$, что $l_{n}>c$ для всех $n \geqslant n_{c}$. Действительно, для любого целого положительного числа $c$ найдется такое целое положительное число $n_{c}$, что каждый элемент группы $B=\left\langle b_{1}\right\rangle \times \cdots$, представимый словом над $\left\{a_{1}^{ \pm 1}, \ldots, a_{d}^{ \pm 1}\right\}$ длины, не превосходящей $c$, содержится в $\left\langle b_{1}\right\rangle \times \cdots \times\left\langle b_{n_{c}-1}\right\rangle$.

Покажем, что найдется целое положительное число $m$, для которого

$$
\left|\bigcup_{1 \leqslant j \leqslant m} \chi_{j}\left(x_{j}^{\tau_{j}}\right)\right|>q r .
$$

Действительно, в противном случае для любого целого положительного числа $m$

$$
\left|\bigcup_{1 \leqslant j \leqslant m} \chi_{j}\left(x_{j}^{\tau_{j}}\right)\right| \leqslant q r
$$

и, следовательно, найдется целое положительное число $c$ такое, что множество $\bigcup_{j \geqslant 1} \chi_{j}\left(x_{j}^{\tau_{j}}\right)$ содержится в шаре радиуса $c$ графа $\Xi_{0}$ с центром $x_{0}$. Однако, как показано выше, существует целое положительное число $n_{c}$ такое, что $l_{n_{c}}>c$ и, следовательно, $\chi_{j}\left(x_{j}^{\tau_{j}}\right)$ при $j=n_{c}$ не содержится в шаре радиуса $c$ графа $\Xi_{0}$ с центром $x_{0}$. Полученное противоречие доказывает (3.1).

Положим $X=\bigcup_{1 \leqslant j \leqslant m} \chi_{j}\left(x_{i_{j}}^{\tau_{j}}\right)$. Тогда найдется целое положительное число $s$ такое, что для каждого $0 \leqslant j \leqslant m$ и произвольных $y_{1}, y_{2} \in \chi_{j}\left(x_{j}^{\tau_{j}}\right)$ справедливо $d_{\Xi_{0}}\left(y_{1}, y_{2}\right) \leqslant s$. Ясно, что для любого $0 \leqslant j \leqslant m$ блоки системы $\tau_{j}$ порождают клики графа $\Xi_{j}^{s}$ (определяемого по графу $\Xi_{j}$ так, как указано в начале $\S 2$ ). Следовательно, при $0 \leqslant j \leqslant m$ неединичные элементы группы $K_{i_{j}}$ переводят 
каждую вершину графа $\Xi_{j}^{s}$ в смежные с ней вершины. Таким образом, при $1 \leqslant$ $j \leqslant m$ любой неединичный элемент группы $\chi_{j} K_{i_{j}} \chi_{j}^{-1}$ автоморфизмов графа $\Xi_{0}^{s}$ также переводит каждую вершину графа $\Xi_{0}^{s}$ в смежную с ней вершину.

Положим $L=\left\langle K_{i_{0}}, \chi_{j} K_{i_{j}} \chi_{j}^{-1}: 1 \leqslant j \leqslant m\right\rangle$. Отметим, что $L$ порождается набором элементов, каждый из которых отображает любую вершину графа $\Xi_{0}^{s}$ в смежную с ней вершину и имеет конечный порядок. Заметим, кроме того, что $X \subseteq L\left(x_{0}\right)$.

Пусть $R:=\left\langle g \in \operatorname{Aut}_{0}\left(\Xi_{0}\right): d_{\Xi_{0}}(y, g(y)) \leqslant s\right.$ для всех $\left.y \in V\left(\Xi_{0}\right)\right\rangle$. Тогда $L \leqslant T(R):=R \cap T\left(\operatorname{Aut}_{0}\left(\Xi_{0}\right)\right)$. Обозначим через $\Sigma$ подграф графа $\Xi_{0}^{s}$, порожденный $R\left(x_{0}\right)$. Тогда $\Sigma$ связен и $R$ индуцирует на $\Sigma$ группу ограниченных автоморфизмов. В частности, $\operatorname{Aut}_{0}(\Sigma)$ - вершинно-транзитивная группа автоморфизмов графа $\Sigma$ и $L^{V(\Sigma)} \leqslant T\left(\operatorname{Aut}_{0}(\Sigma)\right)$. По предложению 2.1 система импримитивности $\tau$ группы $\operatorname{Aut}(\Sigma)$ на $V(\Sigma)$, состоящая из $T\left(\operatorname{Aut}_{0}(\Sigma)\right)$-орбит, имеет конечные блоки и $\operatorname{Aut}_{0}(\Sigma)^{\tau} \cong \mathbb{Z}^{d}$ для некоторого целого неотрицательного числа $d$.

Покажем, что $x_{0}^{\tau}-$ блок импримитивности группы $\operatorname{Aut}\left(\Xi_{0}\right)$ на $V\left(\Xi_{0}\right)$. Поскольку $R \unlhd \operatorname{Aut}\left(\Xi_{0}\right)$, то $V(\Sigma)$ - блок импримитивности группы $\operatorname{Aut}\left(\Xi_{0}\right)$ на $V\left(\Xi_{0}\right)$. Кроме того, $x_{0}^{\tau}=T\left(\operatorname{Aut}_{0}(\Sigma)\right)\left(x_{0}\right)$ - блок импримитивности группы $\operatorname{Aut}(\Sigma)$ на $V(\Sigma)$. Следовательно, $x_{0}^{\tau}-$ блок импримитивности группы $\operatorname{Aut}\left(\Xi_{0}\right)$ на $V\left(\Xi_{0}\right)$.

Поскольку $\Xi_{0}=\Gamma_{G_{i_{0}}, M_{i_{0}}}$ - граф Кэли группы $G_{i_{0}}$, на котором она действует естественным образом, то, обозначая через $H$ стабилизатор в группе $G_{i_{0}}$ блока импримитивности $x_{0}^{\tau}$ группы $\operatorname{Aut}\left(\Xi_{0}\right)$ на $V\left(\Xi_{0}\right)$, имеем $|H|=\left|x_{0}^{\tau}\right|$. Отсюда следует, что $H$ - конечная группа порядка, большего $q r$. Действительно, поскольку $|X|>q r$ (см. (3.1)), $X \subseteq L\left(x_{0}\right)$ (см. выше), $L \subseteq T(R)$ (см. выше) и $T(R)$ индуцирует на $\Sigma$ группу ограниченных автоморфизмов конечного порядка, то

$$
q r<|X| \leqslant\left|L\left(x_{0}\right)\right| \leqslant\left|T(R)\left(x_{0}\right)\right| \leqslant\left|T\left(\operatorname{Aut}_{0}(\Sigma)\right)\left(x_{0}\right)\right|=\left|x_{0}^{\tau}\right|=|H| .
$$

В то же время $K_{i_{0}} \leqslant H$. Действительно, поскольку $K_{i_{0}} \leqslant L$ (по выбору $L$ ), $L \leqslant T(R)$ (см. выше) и $T(R)$ индуцирует на $\Sigma$ группу ограниченных автоморфизмов конечного порядка, то $K_{i_{0}}$ индуцирует на $\Sigma$ группу ограниченных автоморфизмов конечного порядка и, следовательно, оставляет на месте $T\left(\operatorname{Aut}_{0}(\Sigma)\right)$-орбиту $x_{0}^{\tau}$.

Таким образом, группа $G \cong G_{i_{0}} / K_{i_{0}}$ содержит конечную подгруппу $H / K_{i_{0}}$ порядка, большего $r$. Полученное противоречие завершает доказательство теоремы.

ЗАмЕчаниЕ 3.1. Как следует из приведенного доказательства в качестве $G$ в теореме 3.1 может быть взята любая группа, представимая в виде $G=A / B$, где $A$ - группа с системой порождающих $\left\{a_{1}^{ \pm 1}, \ldots, a_{d}^{ \pm 1}\right\}(d$ - целое положительное число) и $B$ - центральная подгруппа группы $A$ такие, что выполнены следующие условия:

1) $a_{k} a_{l}^{-1} \notin B$ для всех $1 \leqslant k \leqslant d, 1 \leqslant l \leqslant d, k \neq l$, и $a_{k} a_{l} \notin B$ для всех $1 \leqslant k \leqslant d, 1 \leqslant l \leqslant d$

2) $B$ - свободная абелева группа счетного ранга; 
3) порядки всех конечных подгрупп группы $A / B$ не превосходят некоторого целого положительного числа $r$.

При этом в качестве $M$ может быть взято множество $\left\{a_{i}^{ \pm 1} B: 1 \leqslant i \leqslant d\right\}$, а в качестве графа $\Delta$ c $|V(\Delta)|=q$, для которого граф $\Gamma_{G, M}$ имеет бесконечное число накрывающих $G$-симметрических расширений посредством $\Delta,-$ произвольный граф Кэли циклической группы порядка $q$ (например, в качестве $\Delta$ может быть взят полный граф с $q$ вершинами).

Приведем примеры группы $A$ с системой порождающих $\left\{a_{1}^{ \pm 1}, \ldots, a_{d}^{ \pm 1}\right\}$, где $d$ - целое положительное число, и центральной подгруппы $B$ группы $A$, для которых выполнены условия 1)-3) из замечания 3.1 (или, что то же, из доказательства теоремы 3.1).

ПримеР 3.1. Покажем, что для произвольных целых чисел $n \geqslant 2$ и $d \geqslant 2$ существуют группа $A$ с системой порождающих $\left\{a_{1}^{ \pm 1}, \ldots, a_{d}^{ \pm 1}\right\}$ и ее центральная подгруппа $B$, которые обладают свойствами 1)-3) из замечания 3.1 и для которых $A / B$ - свободная разрешимая группа ступени $n$ с $d$ (свободными) порождающими. Согласно замечанию 3.1 этим будет доказано, что в качестве группы $G$ в теореме 3.1 можно взять свободную разрешимую группу ступени $n$ с $d$ (свободными) порождающими.

Пусть $F$ - свободная группа со свободной системой порождающих $\left\{f_{1}^{ \pm 1}, \ldots\right.$ $\left.\ldots, f_{d}^{ \pm 1}\right\}$ и $N=F^{(n)}-n$-й член ее ряда коммутантов. Тогда согласно [11, теорема A] (где следует положить $R$ равной $(n-1)$-му члену ряда коммутантов группы $F$ и $c$, равным 2) с учетом формулы Хопфа (см. [11, с. 125]) имеем

$$
N /[N, F]=[F, F] \cap N /[N, F]=C_{1} \times C_{2},
$$

где $C_{1}$ - свободная абелева группа счетного ранга и $C_{2}$ - некоторая абелева группа. Обозначим через $Q$ полный прообраз $C_{2}$ при естественном гомоморфизме $F \rightarrow F /[N, F]$. Положим $A=F / Q, a_{i}=f_{i} Q \in F / Q$ для всех $1 \leqslant i \leqslant d$ и $B=N / Q \cong C_{1}$. Тогда $\left\{a_{1}^{ \pm 1}, \ldots, a_{d}^{ \pm 1}\right\}$ - система порождающих группы $A$ и $B$ - центральная подгруппа группы $A$, причем для них выполняются условия 1 ) и 2) из замечания 3.1. Поскольку $A / B \cong F / N$ - свободная разрешимая группа ступени $n$ с $d$ (свободными) порождающими, то $A / B$ не содержит неединичных элементов конечного порядка и, следовательно, для $A / B$ выполнено условие 3 ) из замечания 3.1. Таким образом, $A,\left\{a_{1}^{ \pm 1}, \ldots, a_{d}^{ \pm 1}\right\}$ и $B$ обладают требуемыми свойствами.

Как показывает пример 3.1, группа $G$ в теореме 3.1 может быть группой без кручения (т. е. без неединичных элементов конечного порядка). С другой стороны, следующий пример показывает, что группа $G$ в теореме 3.1 может быть периодической и даже группой, все собственные подгруппы которой имеют фиксированный простой порядок.

ПримеР 3.2. Пусть $G(\infty)$ - построенная в [12, п. 27.2] бесконечная 2-порожденная группа, все собственные подгруппы которой имеют фиксированный простой порядок. Группа $G(\infty)$ представима в виде $F / N$, где $F$ - свободная группа со свободной системой порождающих $\left\{f_{1}^{ \pm 1}, f_{2}^{ \pm 1}\right\}$ и $N$ - нормальная подгруппа 
группы $F$, построенная в соответствии с [12, п. 27.2]. Согласно [12, леммы 27.2, 25.1 и теорема 31.1] группа $N /[N, F]$ является свободной абелевой группой счетного ранга. Таким образом, полагая $A=F /[N, F], a_{1}=f_{1}[N, F] \in F /[N, F]$, $a_{2}=f_{2}[N, F] \in F /[N, F]$ и $B=N /[N, F]$, получаем, что группа $A$ с системой порождающих $\left\{a_{1}^{ \pm 1}, a_{2}^{ \pm 1}\right\}$ и ее центральная подгруппа $B$ удовлетворяют условиям 1)-3) из замечания 3.1. Согласно замечанию 3.1 в качестве группы $G$ в теореме 3.1 может быть взята группа $A / B \cong G(\infty)$.

ЗАмЕчАНИЕ 3.2. С учетом замечания 3.1 из доказательства теоремы 3.1 и примера 3.2 следует справедливость теоремы 1 из [1], приведенной в [1] без доказательства.

\section{§4. Симметрические и $\operatorname{Aut}_{0}\left(\Lambda^{d}\right)$-симметрические $q$-расширения решеток $\Lambda^{d}$}

\section{1. Условие периодичности для симметрических $q$-расширений} решеток $\Lambda^{d}$. Как было отмечено во введении, в настоящей работе наш подход к вопросам о конечности числа симметрических и $\operatorname{Aut}_{0}\left(\Lambda^{d}\right)$-симметрических расширений $d$-мерной решетки $\Lambda^{d}$ посредством конечного графа основывается на проверке выполнения для таких расширений условия $\left[n_{1}, \ldots, n_{d}\right]$-периодичности и на следующем результате.

Теорема 4.1. Для произвольных иелых чисел $d \geqslant 1, q \geqslant 1, n_{1}, \ldots, n_{d} \geqslant 1$ существует лищь конечное число графов $Г$, являющихся обобщенными симметрическими q-расширениями решетки $\Lambda^{d}$, реализуемыми такими $G, \sigma, \varphi$, что $(\Gamma, \sigma, \varphi)$ удовлетворяет условию $\left[n_{1}, \ldots, n_{d}\right]$-периодичности.

ДокАзАтельство. Пусть $Г$ - обобщенное симметрическое $q$-расширение решетки $\Lambda^{d}$, реализуемое $G, \sigma, \varphi$, причем $(\Gamma, \sigma, \varphi)$ удовлетворяет условию $\left[n_{1}, \ldots, n_{d}\right]$-периодичности. Пусть, кроме того, $\Gamma^{\prime}$ - обобщенное симметрическое $q$-расширение решетки $\Lambda^{d}$, реализуемое $G^{\prime}, \sigma^{\prime}, \varphi^{\prime}$, причем $\left(\Gamma^{\prime}, \sigma^{\prime}, \varphi^{\prime}\right)$ также удовлетворяет условию $\left[n_{1}, \ldots, n_{d}\right]$-периодичности. Положим

$$
\begin{gathered}
X_{0}=\left\{x \in V(\Gamma): 0 \leqslant \operatorname{Pr}_{i}\left(\varphi\left(x^{\sigma}\right)\right)<n_{i} \forall 1 \leqslant i \leqslant d\right\}, \\
X_{j}=\left\{x \in V(\Gamma): \operatorname{Pr}_{j}\left(\varphi\left(x^{\sigma}\right)\right)=n_{j}, 0 \leqslant \operatorname{Pr}_{i}\left(\varphi\left(x^{\sigma}\right)\right)<n_{i} \quad \forall 1 \leqslant i \leqslant d, i \neq j\right\}
\end{gathered}
$$

для каждого $1 \leqslant j \leqslant d$ и

$$
X=\bigcup_{0 \leqslant j \leqslant d} X_{j} .
$$

Аналогично, положим

$$
\begin{gathered}
X_{0}^{\prime}=\left\{x \in V\left(\Gamma^{\prime}\right): 0 \leqslant \operatorname{Pr}_{i}\left(\varphi^{\prime}\left(x^{\sigma^{\prime}}\right)\right)<n_{i} \forall 1 \leqslant i \leqslant d\right\}, \\
X_{j}^{\prime}=\left\{x \in V\left(\Gamma^{\prime}\right): \operatorname{Pr}_{j}\left(\varphi^{\prime}\left(x^{\sigma^{\prime}}\right)\right)=n_{j}, 0 \leqslant \operatorname{Pr}_{i}\left(\varphi^{\prime}\left(x^{\sigma^{\prime}}\right)\right)<n_{i} \quad \forall 1 \leqslant i \leqslant d, i \neq j\right\}
\end{gathered}
$$

для каждого $1 \leqslant j \leqslant d$ и

$$
X^{\prime}=\bigcup_{0 \leqslant j \leqslant d} X_{j}^{\prime} .
$$


Для доказательства теоремы достаточно убедиться в том, что графы $Г$ и $\Gamma^{\prime}$ изоморфны, если выполнены следующие условия:

1) существует изоморфизм $\psi$ подграфа $\langle X\rangle_{\Gamma}$ на подграф $\left\langle X^{\prime}\right\rangle_{\Gamma^{\prime}}$ такой, что для любого $x \in X$ справедливо равенство $\varphi\left(x^{\sigma}\right)=\varphi^{\prime}\left(\psi(x)^{\sigma^{\prime}}\right)$;

2) существуют $g_{1}, \ldots, g_{d} \in \operatorname{Aut}(\Gamma)$ и $g_{1}^{\prime}, \ldots, g_{d}^{\prime} \in \operatorname{Aut}\left(\Gamma^{\prime}\right)$ такие, что:

(i) $\left[g_{k}, g_{l}\right]=1$ и $\left[g_{k}^{\prime}, g_{l}^{\prime}\right]=1$ для всех $1 \leqslant k<l \leqslant d$,

(ii) $\varphi g_{k}^{\sigma} \varphi^{-1}=t_{k}^{n_{k}}=\varphi^{\prime}\left(g_{k}^{\prime}\right)^{\sigma^{\prime}}\left(\varphi^{\prime}\right)^{-1}$ для всех $1 \leqslant k \leqslant d$,

(iii) для любого $1 \leqslant j \leqslant d$ и для любой вершины $x$ графа $Г$ таких, что $\operatorname{Pr}_{j}\left(\varphi\left(x^{\sigma}\right)\right)=0$ и $0 \leqslant \operatorname{Pr}_{i}\left(\varphi\left(x^{\sigma}\right)\right)<n_{i}$ для всех $1 \leqslant i \leqslant d$ с условием $i \neq j$, справедливо равенство $\psi\left(g_{j}(x)\right)=g_{j}^{\prime}(\psi(x))$.

Предположим, что для Г и $\Gamma^{\prime}$ выполнены условия 1), 2). Следующим образом определим отображение $\widetilde{\psi}: V(\Gamma) \rightarrow V\left(\Gamma^{\prime}\right)$. Пусть $x \in V(\Gamma)$. Тогда найдутся однозначно определенные целые числа $k_{1}(x), \ldots, k_{d}(x)$ такие, что $g_{1}^{k_{1}(x)} \cdots g_{d}^{k_{d}(x)}(x) \in X_{0}$. Полагаем

$$
\widetilde{\psi}(x)=\left(g_{1}^{\prime}\right)^{-k_{1}(x)} \cdots\left(g_{d}^{\prime}\right)^{-k_{d}(x)} \psi\left(g_{1}^{k_{1}(x)} \cdots g_{d}^{k_{d}(x)}(x)\right) .
$$

Ясно, что это определение корректно. Кроме того, ясно, что $\widetilde{\psi}-$ биекция $V(\Gamma)$ на $V\left(\Gamma^{\prime}\right)$. Покажем, что $\widetilde{\psi}$ является изоморфизмом графа $Г$ на граф $\Gamma^{\prime}$.

Пусть $x$ и $y$ - некоторые различные вершины графа Г. Покажем, что $\{x, y\} \in E(\Gamma)$ тогда и только тогда, когда $\{\widetilde{\psi}(x), \widetilde{\psi}(y)\} \in E\left(\Gamma^{\prime}\right)$. Очевидно, достаточно рассмотреть случай, когда для некоторого $1 \leqslant j \leqslant d$ имеем $\operatorname{Pr}_{j}\left(\varphi\left(x^{\sigma}\right)\right)=\operatorname{Pr}_{j}\left(\varphi\left(y^{\sigma}\right)\right)+1$ и $\operatorname{Pr}_{i}\left(\varphi\left(x^{\sigma}\right)\right)=\operatorname{Pr}_{i}\left(\varphi\left(y^{\sigma}\right)\right)$ для всех $1 \leqslant i \leqslant d$ с условием $i \neq j$. Заметим, что в этом случае $k_{j}(y) \in\left\{k_{j}(x), k_{j}(x)+1\right\}$ и $k_{i}(x)=k_{i}(y)$ для всех $1 \leqslant i \leqslant d$ с условием $i \neq j$.

Если $k_{j}(y)=k_{j}(x)$, то $\{x, y\} \in E(\Gamma)$ тогда и только тогда, когда

$$
\left\{g_{1}^{k_{1}(x)} \cdots g_{d}^{k_{d}(x)}(x), g_{1}^{k_{1}(x)} \cdots g_{d}^{k_{d}(x)}(y)\right\} \in E(\Gamma),
$$

т. е. тогда и только тогда, когда

$$
\left\{\psi\left(g_{1}^{k_{1}(x)} \cdots g_{d}^{k_{d}(x)}(x)\right), \psi\left(g_{1}^{k_{1}(x)} \cdots g_{d}^{k_{d}(x)}(y)\right)\right\} \in E\left(\Gamma^{\prime}\right),
$$

что, в свою очередь, эквивалентно включению

$$
\begin{aligned}
& \left\{\left(g_{1}^{\prime}\right)^{-k_{1}(x)} \cdots\left(g_{d}^{\prime}\right)^{-k_{d}(x)}\left(\psi\left(g_{1}^{k_{1}(x)} \cdots g_{d}^{k_{d}(x)}(x)\right)\right),\right. \\
& \left.\left(g_{1}^{\prime}\right)^{-k_{1}(x)} \cdots\left(g_{d}^{\prime}\right)^{-k_{d}(x)}\left(\psi\left(g_{1}^{k_{1}(x)} \cdots g_{d}^{k_{d}(x)}(y)\right)\right)\right\} \in E\left(\Gamma^{\prime}\right) .
\end{aligned}
$$

Поскольку здесь

$$
\left(g_{1}^{\prime}\right)^{-k_{1}(x)} \cdots\left(g_{d}^{\prime}\right)^{-k_{d}(x)}\left(\psi\left(g_{1}^{k_{1}(x)} \cdots g_{d}^{k_{d}(x)}(x)\right)\right)=\widetilde{\psi}(x)
$$

и (с учетом того, что в рассматриваемом случае $k_{i}(y)=k_{i}(x)$ для всех $\left.1 \leqslant i \leqslant d\right)$

$$
\left(g_{1}^{\prime}\right)^{-k_{1}(x)} \cdots\left(g_{d}^{\prime}\right)^{-k_{d}(x)}\left(\psi\left(g_{1}^{k_{1}(x)} \cdots g_{d}^{k_{d}(x)}(y)\right)\right)=\widetilde{\psi}(y),
$$


то в рассматриваемом случае $\{x, y\} \in E(\Gamma)$ тогда и только тогда, когда $\{\widetilde{\psi}(x), \widetilde{\psi}(y)\} \in E\left(\Gamma^{\prime}\right)$.

Предположим, что $k_{j}(y)=k_{j}(x)+1$. В этом случае $\{x, y\} \in E(\Gamma)$ тогда и только тогда, когда

$$
\begin{aligned}
\left\{g_{1}^{k_{1}(y)} \cdots g_{d}^{k_{d}(y)}(x), g_{1}^{k_{1}(y)} \cdots\right. & \left.g_{d}^{k_{d}(y)}(y)\right\} \\
& =\left\{g_{j} g_{1}^{k_{1}(x)} \cdots g_{d}^{k_{d}(x)}(x), g_{1}^{k_{1}(y)} \cdots g_{d}^{k_{d}(y)}(y)\right\} \in E(\Gamma) .
\end{aligned}
$$

Поскольку в случае $\{x, y\} \in E(\Gamma)$ с учетом $k_{j}(y)=k_{j}(x)+1$ имеем $\operatorname{Pr}_{j}\left(\varphi\left(g_{1}^{k_{1}(x)} \ldots\right.\right.$ $\left.\left.\cdots g_{d}^{k_{d}(x)}(x)^{\sigma}\right)\right)=0$ и, следовательно, $g_{j} g_{1}^{k_{1}(x)} \cdots g_{d}^{k_{d}(x)}(x) \in X$, то в силу условия 1) включение $\{x, y\} \in E(\Gamma)$ эквивалентно включению

$$
\left\{\psi\left(g_{j} g_{1}^{k_{1}(x)} \cdots g_{d}^{k_{d}(x)}(x)\right), \psi\left(g_{1}^{k_{1}(y)} \cdots g_{d}^{k_{d}(y)}(y)\right)\right\} \in E\left(\Gamma^{\prime}\right) .
$$

Таким образом, согласно условию 2) включение $\{x, y\} \in E(\Gamma)$ эквивалентно включению

$$
\left\{g_{j}^{\prime}\left(\psi\left(g_{1}^{k_{1}(x)} \cdots g_{d}^{k_{d}(x)}(x)\right)\right), \psi\left(g_{1}^{k_{1}(y)} \cdots g_{d}^{k_{d}(y)}(y)\right)\right\} \in E\left(\Gamma^{\prime}\right)
$$

и, следовательно, эквивалентно включению

$$
\begin{gathered}
\left\{\left(g_{1}^{\prime}\right)^{-k_{1}(y)} \cdots\left(g_{d}^{\prime}\right)^{-k_{d}(y)} g_{j}^{\prime}\left(\psi\left(g_{1}^{k_{1}(x)} \cdots g_{d}^{k_{d}(x)}(x)\right)\right),\right. \\
\left.\quad\left(g_{1}^{\prime}\right)^{-k_{1}(y)} \cdots\left(g_{d}^{\prime}\right)^{-k_{d}(y)}\left(\psi\left(g_{1}^{k_{1}(y)} \cdots g_{d}^{k_{d}(y)}(y)\right)\right)\right\} \\
\quad=\left\{\left(g_{1}^{\prime}\right)^{-k_{1}(x)} \cdots\left(g_{d}^{\prime}\right)^{-k_{d}(x)}\left(\psi\left(g_{1}^{k_{1}(x)} \cdots g_{d}^{k_{d}(x)}(x)\right)\right),\right. \\
\left.\quad\left(g_{1}^{\prime}\right)^{-k_{1}(y)} \cdots\left(g_{d}^{\prime}\right)^{-k_{d}(y)}\left(\psi\left(g_{1}^{k_{1}(y)} \cdots g_{d}^{k_{d}(y)}(y)\right)\right)\right\} \\
=\{\widetilde{\psi}(x), \widetilde{\psi}(y)\} \in E\left(\Gamma^{\prime}\right),
\end{gathered}
$$

что завершает доказательство теоремы.

ЗАмЕчАниЕ 4.1. Непосредственным следствием теоремы 4.1 является конечность числа обобщенных симметрических $q$-расширений решетки $\Lambda^{1}$ для произвольного целого положительного числа $q$.

В дальнейшем с использованием теоремы 4.1 мы установим конечность числа (обобщенных) симметрических $q$-расширений решетки $\Lambda^{d}$ в ряде представляющих интерес случаев (см. следствия 4.2-4.5).

Пусть $d$ и $q$ - целые положительные числа, и пусть $\Gamma$ - обобщенное симметрическое $q$-расширение решетки $\Lambda^{d}$, реализуемое некоторыми $G, \sigma, \varphi$. Тогда $\varphi G^{\sigma} \varphi^{-1}$ - вершинно-транзитивная группа автоморфизмов решетки $\Lambda^{d}$. Следовательно, для каждого $1 \leqslant i \leqslant d$ найдется целое положительное число $s$ такое, что $t_{i}^{s} \in \varphi G^{\sigma} \varphi^{-1}$, причем (поскольку стабилизатор вершины решетки $\Lambda^{d}$ в группе $\operatorname{Aut}\left(\Lambda^{d}\right)$ имеет порядок $2^{d} d$ !) число $s$ может быть выбрано не превосходящим $2^{d} d$ !. Таким образом, обозначая для каждого $1 \leqslant i \leqslant d$ через $s_{i}(\Gamma, G, \sigma, \varphi)$ наименьшее из целых положительных чисел $s$ со свойством $t_{i}^{s} \in \varphi G^{\sigma} \varphi^{-1}$, имеем $\left|s_{i}(\Gamma, G, \sigma, \varphi)\right| \leqslant 2^{d} d$ !. Заметим, что если $\operatorname{Aut}_{0}\left(\Lambda^{d}\right) \leqslant \varphi G^{\sigma} \varphi^{-1}$ (в частности, если $\Gamma$ - обобщенное $\operatorname{Aut}_{0}\left(\Lambda^{d}\right)$-симметрическое $q$-расширение решетки $\Lambda^{d}$, реализуемое $G, \sigma, \varphi)$, то $s_{1}(\Gamma, G, \sigma, \varphi)=\cdots=s_{d}(\Gamma, G, \sigma, \varphi)=1$. Обратно, последние равенства влекут, очевидно, $\operatorname{Aut}_{0}\left(\Lambda^{d}\right) \leqslant \varphi G^{\sigma} \varphi^{-1}$. 
Теорема 4.2. Пусть Г - обобщенное симметрическое q-расширение решетки $\Lambda^{d}$ для некоторых целых положительных чисел $d$ и $q$, реализуемое $G$, $\sigma, \varphi$. Предположим, что группа $G$ имеет конечный стабилизатор $G_{x}$ вершины $x$ графа $\Gamma$. Тогда $(\Gamma, \sigma, \varphi)$ удовлетворяет условию $\left[n_{1}, \ldots, n_{d}\right]$-периодичноcmu, где $n_{i} \leqslant s_{i}(\Gamma, G, \sigma, \varphi)\left(q\left|G_{x}\right|\right)^{i-1} \leqslant 2^{d} d !\left(q\left|G_{x}\right|\right)^{i-1}$ для всех $1 \leqslant i \leqslant d$.

ДоказАтельство. Положим $s_{i}=s_{i}(\Gamma, G, \sigma, \varphi), 1 \leqslant i \leqslant d$. Для доказательства теоремы последовательно определим для каждого $1 \leqslant i \leqslant d$ элемент $g_{i}$ группы $G$ такой, что $\left[g_{i}, g_{j}\right]=1$ для всех $1 \leqslant j<i$ и $\varphi g_{i}^{\sigma} \varphi^{-1}=t_{i}^{s_{i} m_{i}}$, где $0<m_{i} \leqslant\left(q\left|G_{x}\right|\right)^{i-1}$. В качестве $g_{1}$ возьмем произвольный элемент группы $G$ со свойством $\varphi g_{1}^{\sigma} \varphi^{-1}=t_{1}^{s_{1}}$. Предположим теперь, что $i>1$ и элементы $g_{1}, \ldots, g_{i-1}$ уже определены. Пусть $a$ - такой элемент группы $G$, что $\varphi a^{\sigma} \varphi^{-1}=t_{i}^{s_{i}}$. Тогда для каждого $1 \leqslant j \leqslant i-1$ и произвольного целого $k$ элемент $\left[g_{j}, a^{k}\right]$ группы $G$ стабилизирует блок $x^{\sigma}$. Однако в группе $G$ имеется лишь $q\left|G_{x}\right|$ элементов, стабилизирующих блок $x^{\sigma}$. Следовательно, $\left[g_{j}, a^{k_{1}}\right]=\left[g_{j}, a^{k_{2}}\right]$ для некоторых $0 \leqslant k_{1}<k_{2} \leqslant q\left|G_{x}\right|$. Поэтому, полагая $m_{j}^{\prime}=k_{2}-k_{1}$, имеем $\left[g_{j}, a^{m_{j}^{\prime}}\right]=1$, где $0<m_{j}^{\prime} \leqslant q\left|G_{x}\right|$. Следовательно, для элемента $g_{i}:=a^{m_{1}^{\prime} \cdots m_{i-1}^{\prime}}$ справедливо равенство $\left[g_{i}, g_{j}\right]=1$ при всех $1 \leqslant j<i$ и $\varphi g_{i}^{\sigma} \varphi^{-1}=t_{i}^{s_{i} m_{i}}$, где $m_{i}:=m_{1}^{\prime} \cdots m_{i-1}^{\prime} \leqslant\left(q\left|G_{x}\right|\right)^{i-1}$.

Таким образом, полагая $n_{i}=s_{i} m_{i}$ для каждого $1 \leqslant i \leqslant d$, получаем, что $(\Gamma, \sigma, \varphi)$ удовлетворяет условию $\left[n_{1}, \ldots, n_{d}\right]$-периодичности, где $n_{i} \leqslant$ $s_{i}(\Gamma, G, \sigma, \varphi)\left(q\left|G_{x}\right|\right)^{i-1}$ для каждого $1 \leqslant i \leqslant d$. Поскольку для каждого $1 \leqslant i \leqslant d$ имеем $\left|s_{i}(\Gamma, G, \sigma, \varphi)\right| \leqslant 2^{d} d$ !, теорема доказана.

Теорема 4.2 влечет справедливость следующего утверждения.

СЛЕДСТВИЕ 4.1. Пусть Г - обобщенное симметрическое q-расширение решетки $\Lambda^{d}$ для некоторых целых положительных чисел $d$ u $q$, реализуемое $G$, $\sigma, \varphi$. Предположим, что группа $G$ имеет конечный стабилизатор $G_{x}$ вериинъ $x$ графа $\Gamma$. Тогда $\Gamma$ допускает группу автоморфизмов, изоморфную $\mathbb{Z}^{d}, c$ не более чем $q\left(2^{d} d !\right)^{d}\left(q\left|G_{x}\right|\right)^{\frac{d(d-1)}{2}}$ орбитами.

Следующее утверждение непосредственно вытекает из теорем 4.1, 4.2.

СлеДСТвиЕ 4.2. Для произвольных иелых положительных чисел $d, q u m$ имеется лищь конечное число обобщенных симметрических q-расширений решетки $\Lambda^{d}$, которые могут быть реализованы такими $G, \sigma, \varphi$, что порядок стабилизатора вершинь в группе $G$ не превосходит $m$.

СлЕДСТвИЕ 4.3. Пусть Г - накрывающее симметрическое q-расширение решетки $\Lambda^{d}$ для некоторых целых положительных чисел $d$ u $q$, реализуемое $G$, $\sigma, \varphi$. Тогда $(\Gamma, \sigma, \varphi)$ удовлетворяет условию $\left[n_{1}, \ldots, n_{d}\right]$-периодичности, где $n_{i} \leqslant\left(2^{d} d !\right)^{i}(q !)^{i-1}$ для всех $1 \leqslant i \leqslant d$. В частности, для произвольных иелых положительных чисел $d$ и q имеется лишь конечное число накрывающих симметрических q-расширений решетки $\Lambda^{d}$.

ДокАЗАТЕЛЬСтво. Пусть $Г$ - произвольное накрывающее симметрическое $q$-расширение решетки $\Lambda^{d}$, где $d$ и $q$ - целые положительные числа, реализуемое некоторыми $G, \sigma, \varphi$. Для $x \in V(\Gamma)$, если элемент $g$ группы $G$ стабилизирует 
поэлементно множество $x^{\sigma}$ и стабилизирует глобально каждый блок системы импримитивности $\sigma$ группы $G$, с учетом связности $Г$ имеем $g=1$. Следовательно, поэлементный стабилизатор множества $x^{\sigma}$ в группе $G$ изоморфно вкладывается в стабилизатор вершины решетки $\Lambda^{d}$ в группе $\operatorname{Aut}\left(\Lambda^{d}\right)$, порядок которого равен $2^{d} d$ !. Таким образом, $\left|G_{x}\right| \leqslant 2^{d} d !(q-1)$ !. Справедливость следствия вытекает теперь из теоремы 4.2 и следствия 4.2.

4.2. $\operatorname{Aut}_{0}\left(\Lambda^{d}\right)$-симметрические $q$-расширения решеток $\Lambda^{d}$ и условие периодичности для них. Далее в этом параграфе рассматриваются $\operatorname{Aut}_{0}\left(\Lambda^{d}\right)$-симметрические $q$-расширения решеток $\Lambda^{d}$, где $d$ и $q$ - целые положительные числа. Как было отмечено во введении, этот случай представляет интерес для физики.

Следующее предложение 4.1, полученное в [1], показывает, в частности, что если $\Gamma-\operatorname{Aut}_{0}\left(\Lambda^{d}\right)$-симметрическое $q$-расширение решетки $\Lambda^{d}$, то соответствующая этому расширению система импримитивности $\sigma$ определена однозначно. Для доказательства этого предложения достаточно воспользоваться применительно к Г предложением 2.2 , полагая в нем $G=\operatorname{Aut}_{0}(\Gamma)$.

ПреДЛОЖенИЕ 4.1. Пусть $Г-\operatorname{Aut}_{0}\left(\Lambda^{d}\right)$-симметрическое расширение решетки $\Lambda^{d}$, где $d$ - целое положительное число, посредством конечного графа, реализуемое $G, \sigma, \varphi$. Тогда блоки $\sigma$ являются $T\left(\operatorname{Aut}_{0}(\Gamma)\right)$-орбитами на $V(\Gamma)$ и Г является $\operatorname{Aut}_{0}\left(\Lambda^{d}\right)$-симметрическим расширением решетки $\Lambda^{d}$, реализуемым $\operatorname{Aut}_{0}(\Gamma), \sigma, \varphi$.

Пусть $\Gamma-\operatorname{Aut}_{0}\left(\Lambda^{d}\right)$-симметрическое $q$-расширение решетки $\Lambda^{d}$, где $d$ и $q-$ целые положительные числа. Тогда разбиение множества $V(\Gamma)$, состоящее из $T\left(\operatorname{Aut}_{0}(\Gamma)\right)$-орбит, назовем системой блоков на $V(\Gamma)$, соответствующей $\operatorname{Aut}_{0}\left(\Lambda^{d}\right)$-симметрическому q-расширению $Г$ решетки $\Lambda^{d}$.

Из предложения 4.1 вытекает справедливость следующего утверждения.

ПреДЛОЖЕНИЕ 4.2. Пустъ граф $\Gamma_{i}, i \in\{1,2\}$, является $\operatorname{Aut}_{0}\left(\Lambda^{d}\right)$-симметрическим q-расширением решетки $\Lambda^{d}$, где $d$ и q-целье положительные числа, и $\sigma_{i}$ - соответствующая ему система блоков на $V\left(\Gamma_{i}\right)$. Тогда если $\varphi-$ изоморфизм графа $\Gamma_{1}$ на граф $\Gamma_{2}$, то $\varphi$ биективно отображает множество блоков системы $\sigma_{1}$ на множество блоков системы $\sigma_{2}$.

ЗАмечАниЕ 4.2. В отличие от $\operatorname{Aut}_{0}\left(\Lambda^{d}\right)$-симметрического расширения решетки $\Lambda^{d}$, где $d$ - целое положительное число, посредством конечного графа, для произвольного симметрического расширения решетки $\Lambda^{d}$, где $d$ - целое положительное число, посредством конечного графа соответствующая этому расширению система импримитивности $\sigma$ определяется, вообще говоря, неоднозначно. (Это имеет непосредственное отношение к замечанию 2.1.) Так, очевидно, уже $\Gamma=\Lambda^{1}$ является $\operatorname{Aut}\left(\Lambda^{1}\right)$-симметрическим 1-расширением решетки $\Lambda^{1}$, реализуемым группой $G_{1}=\operatorname{Aut}(\Gamma)$, системой импримитивности $\sigma_{1}=\{\{i\}: i \in \mathbb{Z}\}$ и изоморфизмом $\varphi_{1}$, отображающим $\{i\}$ в $i$ для каждого $i \in \mathbb{Z}$, и в то же время является $\operatorname{Aut}\left(\Lambda^{1}\right)$-симметрическим 2-расширением решетки $\Lambda^{1}$, реализуемым группой $G_{2}=\left\langle g_{1}, g_{2}\right\rangle$, где $g_{1}$ отображает $i$ в $i+2$ для каждого $i \in \mathbb{Z}$ и $g_{2}$ отображает $i$ в $-i+1$ для каждого $i \in \mathbb{Z}$, системой 
импримитивности $\sigma_{2}=\{\{2 i, 2 i+1\}: i \in \mathbb{Z}\}$ и изоморфизмом $\varphi_{2}$, отображающим $\{2 i, 2 i+1\}$ в $i$ для каждого $i \in \mathbb{Z}$. Конечно, указанный граф Г является также $\operatorname{Aut}_{0}\left(\Lambda^{1}\right)$-симметрическим 1-расширением решетки $\Lambda^{1}$ (реализуемым $\operatorname{Aut}_{0}(\Gamma)$, $\left.\sigma_{1}, \varphi_{1}\right)$, и соответствующая ему система блоков есть $\sigma_{1}$.

Легко также привести примеры $\operatorname{Aut}\left(\Lambda^{d}\right)$-симметрических расширений решетки $\Lambda^{d}, d$ - целое положительное число, посредством конечного графа, не являющихся $\operatorname{Aut}_{0}\left(\Lambda^{d}\right)$-симметрическими расширениями решетки $\Lambda^{d}, d \geqslant 1$, посредством конечного графа. Так, граф $Г$, заданный следующим образом:

$$
\begin{gathered}
V(\Gamma)=\{(i, k): i \in \mathbb{Z}, k \in\{1,2\}\}, \\
E(\Gamma)=\{\{(2 i-1, k),(2 i, k)\}: i \in \mathbb{Z}, k \in\{1,2\}\} \\
\cup\{\{(2 i, k),(2 i+1, l)\}: i \in \mathbb{Z}, k, l \in\{1,2\}\},
\end{gathered}
$$

является $\operatorname{Aut}\left(\Lambda^{1}\right)$-симметрическим 4-расширением $\Lambda^{1}$, но не является $\operatorname{Aut}_{0}\left(\Lambda^{1}\right)$ симметрическим расширением решетки $\Lambda^{1}$ посредством конечного графа. $\mathrm{C}$ другой стороны, существуют $\mathrm{Aut}_{0}\left(\Lambda^{d}\right)$-симметрические расширения решетки $\Lambda^{d}, d$ - целое положительное число, посредством конечного графа, не являющиеся $\operatorname{Aut}\left(\Lambda^{d}\right)$-симметрическими расширениями решетки $\Lambda^{d}, d \geqslant 1$, посредством конечного графа. При $d=1$ в качестве такого графа можно взять, например, граф с множеством вершин

$$
\{(i, k): i \in \mathbb{Z}, k \in\{1,2,3,4\}\}
$$

и множеством ребер

$$
\begin{aligned}
& \{\{(i, 1),(i+1,1)\},\{(i, 1),(i+1,3)\},\{(i, 2),(i+1,1)\},\{(i, 2),(i+1,3)\}, \\
& \quad\{(i, 3),(i+1,2)\},\{(i, 3),(i+1,4)\},\{(i, 4),(i+1,2)\},\{(i, 4),(i+1,4)\}, \\
& \quad\{(i, 1),(i, 3)\},\{(i, 2),(i, 4)\}: i \in \mathbb{Z}\} .
\end{aligned}
$$

Напомним, что группа подстановок называется квазипримитивной, если каждая ее неединичная нормальная подгруппа транзитивна. Заметим, что каждая транзитивная группа подстановок простой степени квазипримитивна.

Tеорема 4.3. Пусть Г - обобщенное $\operatorname{Aut}_{0}\left(\Lambda^{d}\right)$-симметрическое q-расширение решетки $\Lambda^{d}$ для некоторых целых положительных чисел $d u q$, pеализуемое $G, \sigma, \varphi$, причем стабилизатор в $G$ блока из $\sigma$ индуцирует на этом блоке квазипримитивную группу. Тогда $(\Gamma, \sigma, \varphi)$ удовлетворяет условию $\left[n_{1}, \ldots, n_{d}\right]$-периодичности, где $n_{i} \leqslant(q !)^{i-1}$ для всех $1 \leqslant i \leqslant d$.

ДокАЗАТЕЛЬСтво. Для произвольного элемента $\left(i_{1}, \ldots, i_{d}\right)$ группы $\mathbb{Z}^{d}$ положим $B_{\left(i_{1}, \ldots, i_{d}\right)}=\varphi^{-1}\left(\left(i_{1}, \ldots, i_{d}\right)\right)$. Для каждого $1 \leqslant j \leqslant d$ обозначим через $e_{j}$ элемент $(\underbrace{0,0, \ldots, 0}_{j-1}, 1, \underbrace{0, \ldots, 0}_{d-j})$ группы $\mathbb{Z}^{d}$.

Легко видеть, что для произвольных $\left(i_{1}, \ldots, i_{d}\right) \in \mathbb{Z}^{d}$ и $j \in\{1, \ldots, d\}$, полагая $X=B_{\left(i_{1}, \ldots, i_{d}\right)}, Y=B_{\left(i_{1}, \ldots, i_{d}\right)+e_{j}}$, имеем: $G_{X}^{Y}=1$ тогда и только тогда, когда $G_{Y}^{X}=1$. Действительно, с учетом $G_{\{X\}}=G_{\{Y\}}$ и $\left|G_{\{X\}}^{X}\right|=\left|G_{\{Y\}}^{Y}\right|$ имеем

$$
\left|G_{X}^{Y}\right|=\left|G_{\{X\}}^{X \cup Y}\right| /\left|G_{\{X\}}^{X}\right|=\left|G_{\{Y\}}^{X \cup Y}\right| /\left|G_{\{Y\}}^{Y}\right|=\left|G_{Y}^{X}\right|,
$$

и, следовательно, равенства $G_{X}^{Y}=1$ и $G_{Y}^{X}=1$ равносильны. 
Обозначим через $J$ множество $\left\{j \in\{1, \ldots, d\}: G_{B_{0, \ldots, 0}}^{B_{e_{j}}}=1\right\}$, и пусть $\Lambda_{J}^{d}-$ подграф решетки $\Lambda^{d}$, порожденный множеством вершин $\left\{\left(i_{1}, \ldots, i_{d}\right): i_{k}=0\right.$ для всех $k \in\{1, \ldots, d\} \backslash J\}$. Ясно, что при $J \neq \varnothing$ граф $\Lambda_{J}^{d}$ изоморфен $|J|$-мерной решетке $\Lambda^{|J|}$. Обозначим при $J \neq \varnothing$ через $\psi$ изоморфизм графа $\Lambda_{J}^{d}$ на $|J|$-мерную решетку $\Lambda^{|J|}$, отображающий вершину $\left(i_{1}, \ldots, i_{d}\right)$ графа $\Lambda_{J}^{d}$ в вершину $\left(i_{j_{1}}, \ldots, i_{j_{|J|}}\right)$, где $\left\{j_{1}, \ldots, j_{|J|}\right\}=J$ и $j_{1}<\cdots<j_{|J|}$.

Положим $U_{J}=\varphi^{-1}\left(V\left(\Lambda_{J}^{d}\right)\right)$. Тогда с учетом изложенного в предыдущем абзаце имеем $G_{B_{0, \ldots, 0}}^{U_{J}}=1$. Пусть $\Gamma_{J}=\left\langle U_{J}\right\rangle_{\Gamma}-$ подграф графа $\Gamma$, порожденный множеством $U_{J}, G_{J}:=G_{\left\{U_{J}\right\}}^{U_{J}}$ - ограничение на $U_{J}$ стабилизатора $U_{J}$ (глобального) в группе $G, \sigma_{J}:=\left\{X \in \sigma: X \subseteq U_{J}\right\}=\{X \in \sigma$ : $\operatorname{Pr}_{k}(\varphi(X))=0$ для всех $\left.k \in\{1, \ldots, d\} \backslash J\right\}-$ множество содержащихся в $U_{J}$ блоков $\sigma, \varphi_{J}:=\left.\varphi\right|_{\sigma_{J}}$. Ясно, что при $J \neq \varnothing$ граф $\Gamma_{J}$ является обобщенным $\operatorname{Aut}_{0}\left(\Lambda^{|J|}\right)$-симметрическим $q$-расширением решетки $\Lambda^{|J|}$, реализуемым $G_{J}, \sigma_{J}, \psi \varphi_{J}$. При этом в силу $G_{B_{0}, 0}^{U_{J}}=1$ имеем $\left|\left(G_{J}\right)_{x}\right| \leqslant(q-1)$ ! для $x \in V\left(\Gamma_{J}\right)=U_{J}$. Согласно теореме 4.2 отсюда следует, что $\left(\Gamma_{J}, \sigma_{J}, \psi_{J}\right)$ удовлетворяет условию $\left[n_{1}^{\prime}, \ldots, n_{|J|}^{\prime}\right]$-периодичности, где $n_{i}^{\prime} \leqslant\left(q\left|\left(G_{J}\right)_{x}\right|\right)^{i-1} \leqslant(q !)^{i-1}$ для всех $1 \leqslant i \leqslant|J|$.

Если $k \in\{1, \ldots, d\} \backslash J$, то $G_{B_{0}, \ldots, 0}^{B_{e_{k}}} \neq 1$ и, следовательно, с учетом $G_{B_{0, \ldots, 0}}^{B_{e_{k}}} \unlhd$ $G_{\left\{B_{e_{k}}\right\}}^{B_{e_{k}}}$ и квазипримитивности группы $G_{\left\{B_{e_{k}}\right\}}^{B_{e_{k}}}$ группа $G_{B_{0}, \ldots, 0}^{B_{e_{k}}}$ транзитивна. Тогда для произвольного $\left(i_{1}, \ldots, i_{d}\right) \in \mathbb{Z}^{d}$ каждая вершина из $B_{\left(i_{1}, \ldots, i_{d}\right)}$ смежна в графе Г с каждой вершиной из $B_{\left(i_{1}, \ldots, i_{d}\right)+e_{k}}$.

Теперь легко видеть, что $(\Gamma, \sigma, \varphi)$ удовлетворяет условию $\left[n_{1}, \ldots, n_{d}\right]$-периодичности, где $n_{i}=1$ при $i \in\{1, \ldots, d\} \backslash J$ и $n_{i}=n_{l}^{\prime}$ при $i=j_{l} \in J$. Заметим, что в силу $j_{1}<\cdots<j_{|J|}$ при $i=j_{l} \in J$ имеем $i \geqslant l$ и, следовательно, $n_{i}=n_{l}^{\prime} \leqslant(q !)^{l-1} \leqslant(q !)^{i-1}$. Таким образом, теорема доказана.

Из теоремы 4.3 в силу теоремы 4.1 вытекает справедливость следующего утверждения.

СлеДСтвиЕ 4.4. Для произвольных цельх положительных чисел $d$ u $q$ имеется лищь конечное число обобщенных $\operatorname{Aut}_{0}\left(\Lambda^{d}\right)$-симметрических q-расширений решетки $\Lambda^{d}$, которые могут быть реализованы такими $G, \sigma, \varphi$, что стабилизатор в $G$ блока из $\sigma$ индуцирует на этом блоке квазипримитивную zpynny.

Наконец, поскольку транзитивная группа подстановок простой степени является квазипримитивной, то из теоремы 4.3 вытекает справедливость следующего утверждения.

СлЕДСТвИЕ 4.5. Пусть Г - обобщенное $\operatorname{Aut}_{0}\left(\Lambda^{d}\right)$-симметрическое q-расширение решетки $\Lambda^{d}$ для некоторого целого положительного числа $d$ и некоторого целого положительного простого числа $q$, реализуемое $G, \sigma, \varphi$. Тогда $(\Gamma, \sigma, \varphi)$ удовлетворяет условию $\left[n_{1}, \ldots, n_{d}\right]$-периодичности, где $n_{i} \leqslant(q !)^{i-1}$ для всех $1 \leqslant i \leqslant d$. В частности, в силу теоремы 4.1 для произвольного иелого положительного числа $d$ и произвольного челого простого числа $q$ имеется 
лищь конечное число обобщеннъх $\operatorname{Aut}_{0}\left(\Lambda^{d}\right)$-симметрических q-расширений решетки $\Lambda^{d}$.

\section{§5. $\operatorname{Aut}_{0}\left(\Lambda^{d}\right)$-симметрические 2-расширения решеток $\Lambda^{d}$}

Как было отмечено во введении, значительный интерес представляет задача нахождения всех $\operatorname{Aut}_{0}\left(\Lambda^{d}\right)$-симметрических $q$-расширений решетки $\Lambda^{d}$ для фиксированных $d$ и $q$. В настоящем параграфе дается описание $\operatorname{Aut}_{0}\left(\Lambda^{d}\right)$-симметрических 2-расширений решетки $\Lambda^{d}$ для любого целого положительного числа $d$ (см. теорему 5.1 далее). Это позволяет, в частности, указать для любого $d$ число $\operatorname{Aut}_{0}\left(\Lambda^{d}\right)$-симметрических 2-расширений решетки $\Lambda^{d}$ (см. следствие 5.1 далее).

Пусть $d$ - целое положительное число и $\Gamma-\mathrm{Aut}_{0}\left(\Lambda^{d}\right)$-симметрическое 2 -расширение решетки $\Lambda^{d}$, реализуемое $G, \sigma, \varphi$. Граф Г называется насыщенным $\operatorname{Aut}_{0}\left(\Lambda^{d}\right)$-симметрическим 2-расширением решетки $\Lambda^{d}$, если блоки системы $\sigma$ (напомним, что $\sigma$ согласно предложению 4.1 однозначно определяется графом $Г$ ) порождают в Г подграфы, изоморфные полному графу на двух вершинах. (В общем случае для произвольных целых положительных чисел $d$ и $q$ насыщенными $\operatorname{Aut}_{0}\left(\Lambda^{d}\right)$-симметрическими $q$-расширениями решетки $\Lambda^{d}$ называются $\operatorname{Aut}_{0}\left(\Lambda^{d}\right)$-симметрические расширения решетки $\Lambda^{d}$ посредством полного графа на $q$ вершинах.) Для каждого такого графа $\Gamma$ обозначим через $\nu(\Gamma)$ граф, множество вершин которого совпадает с множеством вершин графа $Г$, а множество ребер получается из множества ребер графа Г удалением всех ребер, соединяющих в Г вершины из одного и того же блока системы $\sigma$. Ясно, что так определенный граф $\nu(\Gamma)$ является обобщенным $\operatorname{Aut}_{0}\left(\Lambda^{d}\right)$-симметрическим 2-расширением решетки $\Lambda^{d}$, реализуемым $G, \sigma, \varphi$.

ЗАмечание 5.1. Очевидно, что соответствие $\Gamma \mapsto \nu(\Gamma)$ задает для каждого целого положительного числа $d$ биекцию множества насыщенных $\operatorname{Aut}_{0}\left(\Lambda^{d}\right)$-симметрических 2-расширений решетки $\Lambda^{d}$ на множество обобщенных $\operatorname{Aut}_{0}\left(\Lambda^{d}\right)$ симметрических 2-расширений решетки $\Lambda^{d}$, не являющихся насыщенными.

Легко видеть, что обобщенные $\operatorname{Aut}_{0}\left(\Lambda^{1}\right)$-симметрические 2 -расширения решетки $\Lambda^{1}$ исчерпываются следующими графами $\Gamma_{n}^{1,2}, 1 \leqslant n \leqslant 4$ (верхние индексы 1,2 означают, что $\Gamma_{n}^{1,2}$ являются обобщенными $\operatorname{Aut}_{0}\left(\Lambda^{d}\right)$-симметрическими $q$-расширениями $d$-мерной решетки $\Lambda^{d}$ при $\left.d=1, q=2\right)$.

Для каждого $1 \leqslant n \leqslant 4$

$$
V\left(\Gamma_{n}^{1,2}\right)=\{(i, k): i \in \mathbb{Z}, k \in\{1,2\}\},
$$

и для каждого $1 \leqslant n \leqslant 4$

$$
E\left(\Gamma_{n}^{1,2}\right)=E_{0}\left(\Gamma_{n}^{1,2}\right) \cup E_{1}\left(\Gamma_{n}^{1,2}\right),
$$

где для

$$
\begin{gathered}
D_{0}=\{\{(i, 1),(i, 2)\}: i \in \mathbb{Z}\}, \\
D_{1}=\{\{(i, k),(i+1, l)\}: i \in \mathbb{Z}, k, l \in\{1,2\}\}
\end{gathered}
$$


множества $E_{0}\left(\Gamma_{n}^{1,2}\right)=E\left(\Gamma_{n}^{1,2}\right) \cap D_{0}$ и $E_{1}\left(\Gamma_{n}^{1,2}\right)=E\left(\Gamma_{n}^{1,2}\right) \cap D_{1}$ задаются следующим образом:

$$
\begin{array}{lll}
n=1: & E_{0}\left(\Gamma_{1}^{1,2}\right)=D_{0}, & E_{1}\left(\Gamma_{1}^{1,2}\right)=\{\{(i, k),(i+1, k)\}: i \in \mathbb{Z}, k \in\{1,2\}\} ; \\
n=2: & E_{0}\left(\Gamma_{2}^{1,2}\right)=D_{0}, & E_{1}\left(\Gamma_{2}^{1,2}\right)=D_{1} ; \\
n=3: & E_{0}\left(\Gamma_{3}^{1,2}\right)=\varnothing, & E_{1}\left(\Gamma_{3}^{1,2}\right)=E_{1}\left(\Gamma_{2}^{1,2}\right) \\
n=4: & E_{0}\left(\Gamma_{4}^{1,2}\right)=\varnothing, & E_{1}\left(\Gamma_{4}^{1,2}\right)=E_{1}\left(\Gamma_{1}^{1,2}\right) .
\end{array}
$$

Отметим, что графы $\Gamma_{1}^{1,2}$ и $\Gamma_{2}^{1,2}$ являются насыщенными $\operatorname{Aut}_{0}\left(\Lambda^{1}\right)$-симметрическими 2-расширениями решетки $\Lambda^{1}, \Gamma_{3}^{1,2}=\nu\left(\Gamma_{2}^{1,2}\right), \Gamma_{4}^{1,2}=\nu\left(\Gamma_{1}^{1,2}\right)$. При этом среди графов $\Gamma_{i}^{1,2}, 1 \leqslant i \leqslant 4$, лишь граф $\Gamma_{4}^{1,2}$ несвязен (и, следовательно, не является $\operatorname{Aut}_{0}\left(\Lambda^{1}\right)$-симметрическим 2-расширением решетки $\left.\Lambda^{1}\right)$.

Далее, несложно показать, что обобщенные $\mathrm{Aut}_{0}\left(\Lambda^{2}\right)$-симметрические 2 -расширения решетки $\Lambda^{2}$ исчерпываются следующими графами $\Gamma_{n}^{2,2}, 1 \leqslant n \leqslant 8$ (верхние индексы 2, 2 означают, что $\Gamma_{n}^{2,2}$ являются обобщенными $\operatorname{Aut}_{0}\left(\Lambda^{d}\right)$ симметрическими $q$-расширениями $d$-мерной решетки $\Lambda^{d}$ при $d=2, q=2$ ).

Для каждого $1 \leqslant n \leqslant 8$

$$
V\left(\Gamma_{n}^{1,2}\right)=\{(i, j, k): i, j \in \mathbb{Z}, k \in\{1,2\}\},
$$

и для каждого $1 \leqslant n \leqslant 8$

$$
E\left(\Gamma_{n}^{2,2}\right)=E_{0}\left(\Gamma_{n}^{2,2}\right) \cup E_{1}\left(\Gamma_{n}^{2,2}\right) \cup E_{2}\left(\Gamma_{n}^{2,2}\right),
$$

где для

$$
\begin{gathered}
D_{0}=\{\{(i, j, 1),(i, j, 2)\}: i, j \in \mathbb{Z}\}, \\
D_{1}=\{\{(i, j, k),(i+1, j, l)\}: i, j \in \mathbb{Z}, k, l \in\{1,2\}\}, \\
D_{2}=\{\{(i, j, k),(i, j+1, l)\}: i, j \in \mathbb{Z}, k, l \in\{1,2\}\}
\end{gathered}
$$

множества $E_{0}\left(\Gamma_{n}^{2,2}\right)=E\left(\Gamma_{n}^{2,2}\right) \cap D_{0}, E_{1}\left(\Gamma_{n}^{2,2}\right)=E\left(\Gamma_{n}^{2,2}\right) \cap D_{1}$ и $E_{2}\left(\Gamma_{n}^{2,2}\right)=E\left(\Gamma_{n}^{2,2}\right) \cap$ $D_{2}$ задаются следующим образом:

$$
\begin{array}{ll}
n=1: & E_{0}\left(\Gamma_{1}^{2,2}\right)=D_{0}, \quad E_{1}\left(\Gamma_{1}^{2,2}\right)=\{\{(i, j, k),(i+1, j, k)\}: i, j \in \mathbb{Z}, k \in\{1,2\}\}, \\
& E_{2}\left(\Gamma_{1}^{2,2}\right)=\{\{(i, j, k),(i, j+1, k)\}: i, j \in \mathbb{Z}, k \in\{1,2\}\} ; \\
n=2: \quad & E_{0}\left(\Gamma_{2}^{2,2}\right)=D_{0}, \quad E_{1}\left(\Gamma_{2}^{2,2}\right)=E_{1}\left(\Gamma_{1}^{2,2}\right), \\
& E_{2}\left(\Gamma_{2}^{2,2}\right)=\{\{(i, j, k),(i, j+1, k)\}: i, j \in \mathbb{Z}, k \in\{1,2\}, i \equiv 0(\bmod 2)\} \\
& \cup\{(i, j, 1),(i, j+1,2)\},\{(i, j, 2),(i, j+1,1)\}: i, j \in \mathbb{Z}, i \equiv 1(\bmod 2)\} ; \\
n=3: \quad & E_{0}\left(\Gamma_{3}^{2,2}\right)=D_{0}, \quad E_{1}\left(\Gamma_{3}^{2,2}\right)=E_{1}\left(\Gamma_{1}^{2,2}\right), \quad E_{2}\left(\Gamma_{3}^{2,2}\right)=D_{2} ; \\
n=4: & E_{0}\left(\Gamma_{4}^{2,2}\right)=D_{0}, \quad E_{1}\left(\Gamma_{4}^{2,2}\right)=D_{1}, \quad E_{2}\left(\Gamma_{4}^{2,2}\right)=D_{2} ; \\
n=5: & E_{0}\left(\Gamma_{5}^{2,2}\right)=\varnothing, \quad E_{1}\left(\Gamma_{5}^{2,2}\right)=E_{1}\left(\Gamma_{2}^{2,2}\right), \quad E_{2}\left(\Gamma_{5}^{2,2}\right)=E_{2}\left(\Gamma_{2}^{2,2}\right) ; \\
n=6: & E_{0}\left(\Gamma_{6}^{2,2}\right)=\varnothing, \quad E_{1}\left(\Gamma_{6}^{2,2}\right)=E_{1}\left(\Gamma_{3}^{2,2}\right), \quad E_{2}\left(\Gamma_{6}^{2,2}\right)=E_{2}\left(\Gamma_{3}^{2,2}\right) ; \\
n=7: & E_{0}\left(\Gamma_{7}^{2,2}\right)=\varnothing, \quad E_{1}\left(\Gamma_{7}^{2,2}\right)=E_{1}\left(\Gamma_{4}^{2,2}\right), \quad E_{2}\left(\Gamma_{7}^{2,2}\right)=E_{2}\left(\Gamma_{4}^{2,2}\right) ; \\
n=8: & E_{0}\left(\Gamma_{8}^{2,2}\right)=\varnothing, \quad E_{1}\left(\Gamma_{8}^{2,2}\right)=E_{1}\left(\Gamma_{1}^{2,2}\right), \quad E_{2}\left(\Gamma_{8}^{2,2}\right)=E_{2}\left(\Gamma_{1}^{2,2}\right) .
\end{array}
$$


Отметим, что графы $\Gamma_{i}^{2,2}, 1 \leqslant i \leqslant 4$, являются насыщенными $\operatorname{Aut}_{0}\left(\Lambda^{2}\right)$ симметрическими 2-расширениями решетки $\Lambda^{2}, \Gamma_{5}^{2,2}=\nu\left(\Gamma_{2}^{2,2}\right), \Gamma_{6}^{2,2}=\nu\left(\Gamma_{3}^{2,2}\right)$, $\Gamma_{7}^{2,2}=\nu\left(\Gamma_{4}^{2,2}\right), \Gamma_{8}^{2,2}=\nu\left(\Gamma_{1}^{2,2}\right)$. При этом среди графов $\Gamma_{i}^{2,2}, 1 \leqslant i \leqslant 8$, лишь граф $\Gamma_{8}^{2,2}$ несвязен (и, следовательно, не является $\operatorname{Aut}_{0}\left(\Lambda^{2}\right)$-симметрическим 2-расширением решетки $\left.\Lambda^{2}\right)$.

Перейдем к описанию $\operatorname{Aut}_{0}\left(\Lambda^{d}\right)$-симметрических 2-расширений решетки $\Lambda^{d}$ для произвольного целого положительного числа $d$.

Пусть $\Gamma$ - произвольное насыщенное $\operatorname{Aut}_{0}\left(\Lambda^{d}\right)$-симметрическое 2-расширение решетки $\Lambda^{d}$, где $d \geqslant 1$, реализуемое некоторыми $G, \sigma, \varphi$. (Согласно предложению 4.1 система импримитивности $\sigma$ группы $G$ совпадает с множеством всех $T\left(\operatorname{Aut}_{0}(\Gamma)\right)$-орбит на $V(\Gamma)$ и можно считать, что $G=\operatorname{Aut}_{0}(\Gamma)$.)

Для целого числа $i, 1 \leqslant i \leqslant d$, скажем, что в графе Г относительно $\varphi$ направление $\{i\}$ реализует тип $\Gamma_{1}^{1,2}$ (соответственно, $\Gamma_{2}^{1,2}$ ), если подграф графа Г, порожденный множеством

$$
\left\{x \in V(\Gamma): \operatorname{Pr}_{j}\left(\varphi\left(x^{\sigma}\right)\right)=0 \quad \forall j \in\{1, \ldots, d\} \backslash\{i\}\right\}
$$

изоморфен графу $\Gamma_{1}^{1,2}$ (соответственно, $\left.\Gamma_{2}^{1,2}\right)$.

Для различных целых чисел $i$ и $j, 1 \leqslant i, j \leqslant d$, скажем, что в графе $\Gamma$ относительно ч пара направлений $\{i, j\}$ реализует тип $\Gamma_{1}^{2,2}$ (соответственно, $\Gamma_{2}^{2,2}, \Gamma_{3}^{2,2}$ или $\left.\Gamma_{4}^{2,2}\right)$, если подграф графа $\Gamma$, порожденный множеством

$$
\left\{x \in V(\Gamma): \operatorname{Pr}_{k}\left(\varphi\left(x^{\sigma}\right)\right)=0 \quad \forall k \in\{1, \ldots, d\} \backslash\{i, j\}\right\}
$$

изоморфен графу $\Gamma_{1}^{2,2}$ (соответственно, $\Gamma_{2}^{2,2}, \Gamma_{3}^{2,2}$ или $\left.\Gamma_{4}^{2,2}\right)$.

Пусть вновь $\Gamma$ - насыщенное $\operatorname{Aut}_{0}\left(\Lambda^{d}\right)$-симметрическое 2-расширение решетки $\Lambda^{d}$, где $d \geqslant 1$, реализуемое некоторыми $G, \sigma, \varphi$. Предположим, что существует целое число $c, 0 \leqslant c \leqslant d$, такое, что для каждого целого числа $i$ с условием $1 \leqslant i \leqslant c$ относительно изоморфизма $\varphi$ в графе $\Gamma$ направление $\{i\}$ реализует тип $\Gamma_{2}^{1,2}$, а для каждого целого числа $i$ с условием $c<i \leqslant d$ относительно изоморфизма $\varphi$ в графе $\Gamma$ направление $\{i\}$ реализует тип $\Gamma_{1}^{1,2}$. Полагая $\mathcal{P}:=\{\{i, j\}: c<i<j \leqslant d$ и относительно изоморфизма $\varphi$ пара направлений $\{i, j\}$ реализует тип $\left.\Gamma_{1}^{2,2}\right\}$, скажем, что в этом случае граф $Г$ относительно изоморфизма ч реализует набор $(c, \mathcal{P})$.

ЗАмечание 5.2. Множество $\mathcal{P}$ совпадает с $\{\{i, j\}: 1 \leqslant i<j \leqslant d$ и относительно изоморфизма $\varphi$ пара направлений $\{i, j\}$ реализует тип $\left.\Gamma_{1}^{2,2}\right\}$, а множество $\{\{i, j\}: c<i<j \leqslant d\} \backslash \mathcal{P}$ совпадает с $\{\{i, j\}: 1 \leqslant i<j \leqslant d$ и относительно изоморфизма $\varphi$ пара направлений $\{i, j\}$ реализует тип $\left.\Gamma_{2}^{2,2}\right\}$. Кроме того, $\{\{i, j\}: 1 \leqslant i \leqslant c<j \leqslant d\}=\{\{i, j\}: 1 \leqslant i<j \leqslant d$ и относительно изоморфизма $\varphi$ пара направлений $\{i, j\}$ реализует тип $\left.\Gamma_{3}^{2,2}\right\}$ и $\{\{i, j\}: 1 \leqslant i<j \leqslant c\}=\{\{i, j\}: 1 \leqslant i<j \leqslant d$ и относительно изоморфизма $\varphi$ пара направлений $\{i, j\}$ реализует тип $\left.\Gamma_{4}^{2,2}\right\}$. 
Далее, скажем, что для целого числа $c$ с условием $0 \leqslant c \leqslant d$ и $\mathcal{P} \subseteq$ $\{\{i, j\}: c<i<j \leqslant d\}$ граф $\Gamma$, являющийся насыщенным $\operatorname{Aut}_{0}\left(\Lambda^{d}\right)$-симметрическим 2-расширением решетки $\Lambda^{d}$, реализует набор $(c, \mathcal{P})$, если для некоторого изоморфизма $\varphi$ графа $\Gamma / \sigma$, где $\sigma$ - соответствующая $\Gamma$ система блоков, на решетку $\Lambda^{d}$ граф $\Gamma$ реализует набор $(c, \mathcal{P})$ относительно $\varphi$.

Следующая теорема дает описание $\operatorname{Aut}_{0}\left(\Lambda^{d}\right)$-симметрических 2-расширений решетки $\Lambda^{d}$ для произвольного целого положительного числа $d$.

Теорема 5.1. Для произвольного целого положительного числа d справедливы следующие утверждения:

1) каждое насыщенное $\operatorname{Aut}_{0}\left(\Lambda^{d}\right)$-симметрическое 2 -расширение решетки $\Lambda^{d}$ реализует некоторый набор $(c, \mathcal{P})$, где $0 \leqslant c \leqslant d u \mathcal{P} \subseteq\{\{i, j\}: c<i<j \leqslant d\}$;

2) для любых $0 \leqslant c \leqslant d u \mathcal{P} \subseteq\{\{i, j\}: c<i<j \leqslant d\}$ существует единственное с точностью до изоморфизма насыщенное $\operatorname{Aut}_{0}\left(\Lambda^{d}\right)$-симметрическое 2-расширение решетки $\Lambda^{d}$, реализующее набор $(c, \mathcal{P})$;

3) граф, реализующий набор $(c, \mathcal{P})$, где $0 \leqslant c \leqslant d u \mathcal{P} \subseteq\{\{i, j\}: c<i<$ $j \leqslant d\}$, изоморфен графу, реализующему набор $\left(c^{*}, \mathcal{P}^{*}\right)$, где $0 \leqslant c^{*} \leqslant d u \mathcal{P}^{*} \subseteq$ $\left\{\{i, j\}: c^{*}<i<j \leqslant d\right\}$, тогда и только тогда, когда $c^{*}=c$ и существует подстановка на $\{c+1, \ldots, d\}$, переводящая $\mathcal{P}$ в $\mathcal{P}^{*}$;

4) если $Г$ - произвольное насъщенное $\operatorname{Aut}_{0}\left(\Lambda^{d}\right)$-симметрическое 2-расширение решетки $\Lambda^{d}$, реализующее отличный от $(0,\{\{i, j\}: 1 \leqslant i<j \leqslant d\})$ набор, то сопоставление графу $Г$ графа $\nu(\Gamma)$ есть сохраняющая отношение изоморфности биекция множества насыщеннъх $\operatorname{Aut}_{0}\left(\Lambda^{d}\right)$-симметрических 2-расширений решетки $\Lambda^{d}$, реализующих отличные от $(0,\{\{i, j\}: 1 \leqslant i<$ $j \leqslant d\})$ наборы, на множество $\operatorname{Aut}_{0}\left(\Lambda^{d}\right)$-симметрических 2-расширений решетки $\Lambda^{d}$, не являющихся насыщенными.

ЗАмечАниЕ 5.3. Утверждение 2) теоремы 5.1 в части единственности (с точностью до изоморфизма) реализации набора $(c, \mathcal{P})$ следует из утверждения 3 ) теоремы 5.1. Однако утверждение 2) в приведенной выше форме доказывается нами индуктивно и используется при доказательстве утверждения 3).

ДокАЗАТЕЛЬСтво теоремы 5.1. Докажем утверждение 1). Пусть Г - произвольное насыщенное $\operatorname{Aut}_{0}\left(\Lambda^{d}\right)$-симметрическое 2-расширение решетки $\Lambda^{d}$, peализуемое некоторыми $G, \sigma, \varphi$. (При этом, см. предложение $4.1, \sigma$ совпадает с множеством $T\left(\operatorname{Aut}_{0}(\Gamma)\right)$-орбит на $V(\Gamma)$.)

Предположим, что ни для какого целого $i$ с условием $1 \leqslant i \leqslant d$ в графе $\Gamma$ относительно $\varphi$ направление $\{i\}$ не реализует тип $\Gamma_{2}^{1,2}$. Тогда, полагая $\mathcal{P}=\{\{i, j\}: 1 \leqslant i<j \leqslant d$ и относительно $\varphi$ пара направлений $\{i, j\}$ реализует тип $\left.\Gamma_{1}^{2,2}\right\}$, получаем, что граф $Г$ является $\operatorname{Aut}_{0}\left(\Lambda^{d}\right)$-симметрическим 2-расширением графа $\Lambda^{d}$ и относительно изоморфизма $\varphi$ реализует набор $(0, \mathcal{P})$.

Предположим теперь, что в графе Г относительно $\varphi$ некоторое направление реализует тип $\Gamma_{2}^{1,2}$. Пусть $i_{1}, \ldots, i_{c}-$ все такие $i, 1 \leqslant i \leqslant d$, что относительно $\varphi$ 
в графе $\Gamma$ направление $\{i\}$ реализует тип $\Gamma_{2}^{1,2}$. Пусть, кроме того, $\psi$ - автоморфизм $\Lambda^{d}$ такой, что $\left\{\psi t_{i_{1}} \psi^{-1}, \ldots, \psi t_{i_{c}} \psi^{-1}\right\}=\left\{t_{1}, \ldots, t_{c}\right\}$. Тогда относительно $\psi \varphi$ в графе $\Gamma$ направления $\{i\}$, где $1 \leqslant i \leqslant c$, реализуют тип $\Gamma_{2}^{1,2}$, а направления $\{i\}$, где $c<i \leqslant d$, реализуют тип $\Gamma_{1}^{1,2}$. Полагая $\mathcal{P}=\{\{i, j\}: c<i<j \leqslant d$ и относительно $\psi \varphi$ пара направлений $\{i, j\}$ реализует тип $\left.\Gamma_{1}^{2,2}\right\}$, получаем, что граф $Г$ является насыщенным $\operatorname{Aut}_{0}\left(\Lambda^{d}\right)$-симметрическим 2-расширением графа $\Lambda^{d}$ и относительно изоморфизма $\psi \varphi$ реализует набор $(c, \mathcal{P})$. Таким образом, утверждение 1) доказано.

Докажем утверждение 2). Очевидно, оно справедливо при $d \leqslant 2$. Предположим, что утверждение 2) справедливо при $d=d_{0} \geqslant 2$. Докажем его справедливость при $d=d_{0}+1$. Пусть $1 \leqslant c \leqslant d_{0}+1$ и $\mathcal{P} \subseteq\left\{\{i, j\}: c<i<j \leqslant d_{0}+1\right\}$.

Если $c=d_{0}+1$, то $\mathcal{P}=\varnothing$ и, очевидно, единственным (с точностью до изоморфизма) насыщенным $\operatorname{Aut}_{0}\left(\Lambda^{d_{0}+1}\right)$-симметрическим 2-расширением решетки $\Lambda^{d_{0}+1}$, реализующим набор $(c, \mathcal{P})$, является граф $\Gamma$, определяемый следующим образом:

$$
\begin{gathered}
V(\Gamma)=\left\{\left(i_{1}, \ldots, i_{d_{0}+1}, k\right): i_{1}, \ldots, i_{d_{0}+1} \in \mathbb{Z}, k \in\{1,2\}\right\}, \\
E(\Gamma)=\left\{\left\{\left(i_{1}, \ldots, i_{d_{0}+1}, 1\right),\left(i_{1}, \ldots, i_{d_{0}+1}, 2\right)\right\}: i_{1}, \ldots, i_{d_{0}+1} \in \mathbb{Z}\right\} \\
\cup\left\{\left\{\left(i_{1}, \ldots, i_{d_{0}+1}, k\right),\left(i_{1}^{\prime}, \ldots, i_{d_{0}+1}^{\prime}, l\right)\right\}: i_{1}, \ldots, i_{d_{0}+1}, i_{1}^{\prime}, \ldots, i_{d_{0}+1}^{\prime} \in \mathbb{Z},\right. \\
\left.k, l \in\{1,2\},\left|i_{1}-i_{1}^{\prime}\right|+\cdots+\left|i_{d_{0}+1}-i_{d_{0}+1}^{\prime}\right|=1\right\} .
\end{gathered}
$$

Пусть $c<d_{0}+1$. По предположению индукции существует единственное (с точностью до изоморфизма) $\operatorname{Aut}_{0}\left(\Lambda^{d_{0}}\right)$-симметрическое 2 -расширение $\Gamma^{\prime}$ решетки $\Lambda^{d_{0}}$, реализующее набор $\left(c, \mathcal{P}^{\prime}\right)$, где $\mathcal{P}^{\prime}=\mathcal{P} \cap\left\{\{i, j\}: c<i<j \leqslant d_{0}\right\}$. Пусть $\Gamma^{\prime}$ реализуется $G^{\prime}, \sigma^{\prime}, \varphi^{\prime}$ и реализует относительно $\varphi^{\prime}$ набор $\left(c, \mathcal{P}^{\prime}\right)$. Отождествим множество вершин $\Gamma^{\prime}$ с множеством $\left\{\left(i_{1}, \ldots, i_{d_{0}}, k\right): i_{1}, \ldots, i_{d_{0}} \in \mathbb{Z}, k \in\right.$ $\{1,2\}\}$ так, чтобы $\varphi^{\prime}\left(\left(i_{1}, \ldots, i_{d_{0}}, k\right)^{\sigma^{\prime}}\right)=\left(i_{1}, \ldots, i_{d_{0}}\right)$ для любых $i_{1}, \ldots, i_{d_{0}} \in \mathbb{Z}$ и $k \in\{1,2\}$.

Определим следующим образом граф Г. Положим

$$
V(\Gamma)=\left\{\left(i_{1}, \ldots, i_{d_{0}+1}, k\right): i_{1}, \ldots, i_{d_{0}+1} \in \mathbb{Z}, k \in\{1,2\}\right\} .
$$

Кроме того, положим

$$
\begin{aligned}
E(\Gamma)= & \left\{\left(i_{1}, \ldots, i_{d_{0}+1}, k\right),\left(i_{1}^{\prime}, \ldots, i_{d_{0}+1}^{\prime}, l\right)\right\}: i_{1}, \ldots, i_{d_{0}+1}, i_{1}^{\prime}, \ldots, i_{d_{0}+1}^{\prime} \in \mathbb{Z}, \\
& \left.k, l \in\{1,2\}, i_{d_{0}+1}=i_{d_{0}+1}^{\prime},\left\{\left(i_{1}, \ldots, i_{d_{0}}, k\right),\left(i_{1}^{\prime}, \ldots, i_{d_{0}}^{\prime}, l\right)\right\} \in E\left(\Gamma^{\prime}\right)\right\} \cup E^{\prime},
\end{aligned}
$$

где $E^{\prime}$ определяется следующим образом:

$$
\begin{gathered}
E^{\prime}=\left\{\left\{\left(i_{1}, \ldots, i_{d_{0}}, i_{d_{0}+1}, k\right),\left(i_{1}, \ldots, i_{d_{0}}, i_{d_{0}+1}+1, l\right)\right\}: i_{1}, \ldots, i_{d_{0}}, i_{d_{0}+1} \in \mathbb{Z},\right. \\
k, l \in\{1,2\}, k=l \text { при } R=\varnothing \text { или при } \sum_{r \in R} i_{r} \equiv 0(\bmod 2), \\
\left.k \neq l \text { при } R \neq \varnothing \text { и } \sum_{r \in R} i_{r} \equiv 1(\bmod 2)\right\}
\end{gathered}
$$

для $R=\left\{r: c<r<d_{0}+1,\left\{r, d_{0}+1\right\} \notin \mathcal{P}\right\}$. 
Покажем, что граф Г является $\operatorname{Aut}_{0}\left(\Lambda^{d_{0}+1}\right)$-симметрическим 2-расширением решетки $\Lambda^{d_{0}+1}$, реализующим набор $(c, \mathcal{P})$.

Предварительно определим автоморфизмы $h_{i}, 0 \leqslant i \leqslant d_{0}$, графа $\Gamma^{\prime}$. Пусть $h_{0}$ - автоморфизм графа $\Gamma^{\prime}$, меняющий местами вершины во всех блоках $\sigma^{\prime}$. Кроме того, для каждого $0<i \leqslant d_{0}$ пусть $h_{i}$ - некоторый автоморфизм графа $\Gamma^{\prime}$ такой, что $\varphi^{\prime} h_{i}^{\sigma^{\prime}}\left(\varphi^{\prime}\right)^{-1}$ есть сдвиг $t_{i}$ решетки $\Lambda^{d_{0}}$.

Теперь следующим образом определим ограниченные автоморфизмы $g_{i}, 0 \leqslant$ $i \leqslant d_{0}+1$, графа Г. Положим

$g_{0}:\left(i_{1}, \ldots, i_{d_{0}+1}, k\right) \mapsto\left(i_{1}, \ldots, i_{d_{0}+1}, l\right), \quad i_{1}, \ldots, i_{d_{0}+1} \in \mathbb{Z}, \quad k, l \in\{1,2\}, \quad k \neq l$.

Если $i \leqslant c$ или $\left\{i, d_{0}+1\right\} \in \mathcal{P}$, то положим

$$
\begin{gathered}
g_{i}:\left(i_{1}, \ldots, i_{d_{0}+1}, k\right) \mapsto\left(i_{1}^{\prime}, \ldots, i_{d_{0}+1}^{\prime}, l\right), \\
i_{1}, \ldots, i_{d_{0}+1}, i_{1}^{\prime}, \ldots, i_{d_{0}+1}^{\prime} \in \mathbb{Z}, \quad k, l \in\{1,2\}, \\
i_{d_{0}+1}=i_{d_{0}+1}^{\prime}, \quad h_{i}\left(\left(i_{1}, \ldots, i_{d_{0}}, k\right)\right)=\left(i_{1}^{\prime}, \ldots, i_{d_{0}}^{\prime}, l\right) .
\end{gathered}
$$

Если $c<i<d_{0}+1$ и $\left\{i, d_{0}+1\right\} \notin \mathcal{P}$, то положим

$$
\begin{gathered}
g_{i}:\left(i_{1}, \ldots, i_{d_{0}+1}, k\right) \mapsto\left(i_{1}^{\prime}, \ldots, i_{d_{0}+1}^{\prime}, l\right), \\
i_{1}, \ldots, i_{d_{0}+1}, i_{1}^{\prime}, \ldots, i_{d_{0}+1}^{\prime} \in \mathbb{Z}, \quad k, l \in\{1,2\}, \\
i_{d_{0}+1}=i_{d_{0}+1}^{\prime}, \quad h_{i}\left(\left(i_{1}, \ldots, i_{d_{0}}, k\right)\right)=\left(i_{1}^{\prime}, \ldots, i_{d_{0}}^{\prime}, l\right) \quad \text { при } \quad i_{d_{0}+1} \equiv 0(\bmod 2), \\
h_{0} h_{i}\left(\left(i_{1}, \ldots, i_{d_{0}}, k\right)\right)=\left(i_{1}^{\prime}, \ldots, i_{d_{0}}^{\prime}, l\right) \quad \text { при } \quad i_{d_{0}+1} \equiv 1(\bmod 2) .
\end{gathered}
$$

Наконец, положим

$$
\begin{gathered}
g_{d_{0}+1}:\left(i_{1}, \ldots, i_{d_{0}}, i_{d_{0}+1}, k\right) \mapsto\left(i_{1}, \ldots, i_{d_{0}}, i_{d_{0}+1}+1, k\right), \\
i_{1}, \ldots, i_{d_{0}+1} \in \mathbb{Z}, \quad k \in\{1,2\} .
\end{gathered}
$$

Из определений автоморфизмов $g_{i}, 0 \leqslant i \leqslant d_{0}+1$, следует, что $G:=\left\langle g_{0}, g_{1}, \ldots\right.$ $\left.\ldots, g_{d_{0}+1}\right\rangle$ - вершинно-транзитивная группа ограниченных автоморфизмов графа $Г$ и $\sigma:=\left\{\left\{\left(i_{1}, \ldots, i_{d_{0}+1}, 1\right),\left(i_{1}, \ldots, i_{d_{0}+1}, 2\right)\right\}: i_{1}, \ldots, i_{d_{0}+1} \in \mathbb{Z}\right\}$ - система импримитивности группы $G$ с блоками порядка 2 , причем

$$
\varphi:\left(\left\{\left(i_{1}, \ldots, i_{d_{0}+1}, 1\right),\left(i_{1}, \ldots, i_{d_{0}+1}, 2\right)\right\}\right) \mapsto\left(i_{1}, \ldots, i_{d_{0}+1}\right), \quad i_{1}, \ldots, i_{d_{0}+1} \in \mathbb{Z},
$$

является изоморфизмом графа $\Gamma / \sigma$ на решетку $\Lambda^{d_{0}+1}$, для которого $\varphi G^{\sigma} \varphi^{-1}=$ $\operatorname{Aut}_{0}\left(\Lambda^{d_{0}+1}\right)$. Следовательно, Г является $\operatorname{Aut}_{0}\left(\Lambda^{d_{0}+1}\right)$-симметрическим 2-расширением решетки $\Lambda^{d_{0}+1}$. При этом из построения графа $Г$ следует, что он относительно $\varphi$ реализует набор $(c, \mathcal{P})$.

Пусть теперь $\Gamma$ - произвольное $\operatorname{Aut}_{0}\left(\Lambda^{d}\right)$-симметрическое 2-расширение решетки $\Lambda^{d}$, где $d=d_{0}+1$, реализуемое некоторыми $G, \sigma, \varphi$ и реализующее набор $(c, \mathcal{P})$ относительно изоморфизма $\varphi$. (При этом, см. предложение $4.1, \sigma$ совпадает с множеством $T\left(\operatorname{Aut}_{0}(\Gamma)\right)$-орбит на $V(\Gamma)$.) Покажем, что этими условиями 
граф Г однозначно (с точностью до изоморфизма) определен. Очевидно, не теряя общности, можно считать, что справедливо следующее утверждение:

(a) $V(\Gamma)=\left\{\left(i_{1}, \ldots, i_{d}, k\right): i_{1}, \ldots, i_{d} \in \mathbb{Z}, k \in\{1,2\}\right\}$,

$$
\begin{aligned}
& \sigma=\left\{\left\{\left(i_{1}, \ldots, i_{d}, 1\right),\left(i_{1}, \ldots, i_{d}, 2\right)\right\}: i_{1}, \ldots, i_{d} \in \mathbb{Z}\right\}, \\
& \varphi: V(\Gamma / \sigma) \rightarrow V\left(\Lambda^{d}\right), \quad \varphi\left(\left\{\left(i_{1}, \ldots, i_{d}, 1\right),\left(i_{1}, \ldots, i_{d}, 2\right)\right\}\right)=\left(i_{1}, \ldots, i_{d}\right) .
\end{aligned}
$$

Предположим сначала, что $c \geqslant 1$. Тогда для каждого целого числа $i_{0}$ подграф графа $\Gamma$, порожденный множеством вершин $\left\{\left(i_{0}, i_{1}, \ldots, i_{d_{0}}, k\right): i_{1}, \ldots\right.$ $\left.\ldots, i_{d_{0}} \in \mathbb{Z}, k \in\{1,2\}\right\}$, является, очевидно, $\operatorname{Aut}_{0}\left(\Lambda^{d_{0}}\right)$-симметрическим 2 -расширением решетки $\Lambda^{d_{0}}$, реализующим набор $\left(c-1, \mathcal{P}^{\prime \prime}\right)$, где $\mathcal{P}^{\prime \prime}=\{\{i, j\}$ : $\left.1 \leqslant i<j \leqslant d_{0},\{i+1, j+1\} \in \mathcal{P}\right\}$. По предположению индукции существует единственное (с точностью до изоморфизма) $\operatorname{Aut}_{0}\left(\Lambda^{d_{0}}\right)$-симметрическое 2 -расширение $\Gamma^{\prime \prime}$ решетки $\Lambda^{d_{0}}$, реализующее набор $\left(c-1, \mathcal{P}^{\prime \prime}\right)$. Пусть $\Gamma^{\prime \prime}$ реализуется $G^{\prime \prime}, \sigma^{\prime \prime}, \varphi^{\prime \prime}$ и реализует относительно $\varphi^{\prime \prime}$ набор $\left(c-1, \mathcal{P}^{\prime \prime}\right)$. Отождествим множество вершин $\Gamma^{\prime \prime}$ с множеством $\left\{\left(i_{1}, \ldots, i_{d_{0}}, k\right): i_{1}, \ldots, i_{d_{0}} \in \mathbb{Z}, k \in\{1,2\}\right\}$ так, чтобы $\varphi^{\prime \prime}\left(\left(i_{1}, \ldots, i_{d_{0}}, k\right)^{\sigma^{\prime \prime}}\right)=\left(i_{1}, \ldots, i_{d_{0}}\right)$ для любых $i_{1}, \ldots, i_{d_{0}} \in \mathbb{Z}$ и $k \in\{1,2\}$. Очевидно, не теряя общности, можно считать, что для каждого целого числа $i$ отображение

$$
\psi_{i}: V\left(\Gamma^{\prime \prime}\right) \rightarrow V(\Gamma), \quad \psi_{i}\left(\left(i_{1}, \ldots, i_{d-1}, k\right)\right)=\left(i, i_{1}, \ldots, i_{d-1}, k\right),
$$

есть изоморфное вложение графа $\Gamma^{\prime \prime}$ в граф Г. Теперь

$$
E(\Gamma)=\left\{\left\{\psi_{i}(x), \psi_{i}(y)\right\}: i \in \mathbb{Z},\{x, y\} \in E\left(\Gamma^{\prime \prime}\right)\right\} \cup E^{\prime \prime},
$$

где

$$
E^{\prime \prime}=\left\{\left\{\left(i_{1}, \ldots, i_{d-1}, i_{d}, k\right),\left(i_{1}, \ldots, i_{d-1}, i_{d}+1, l\right)\right\}: i_{1}, \ldots, i_{d} \in \mathbb{Z}, k, l \in\{1,2\}\right\},
$$

и, следовательно, $\operatorname{Aut}_{0}\left(\Lambda^{d}\right)$-симметрическое 2-расширение решетки $\Lambda^{d}$, реализующее набор $(c, \mathcal{P})$, единственно (с точностью до изоморфизма).

Предположим поэтому, что $c=0$. Очевидно, не теряя общности, можно считать, что справедливо следующее утверждение:

(b) для каждого целого числа $i$ отображение $\varphi_{i}: V\left(\Gamma^{\prime}\right) \rightarrow V(\Gamma), \varphi_{i}\left(\left(i_{1}, \ldots\right.\right.$ $\left.\left.\ldots, i_{d-1}, k\right)\right)=\left(i_{1}, \ldots, i_{d-1}, i, k\right)$, есть изоморфное вложение графа $\Gamma^{\prime}$ в граф $\Gamma$.

Теперь

$$
E(\Gamma)=\left\{\left\{\varphi_{i}(x), \varphi_{i}(y)\right\}: i \in \mathbb{Z},\{x, y\} \in E\left(\Gamma^{\prime}\right)\right\} \cup E^{\prime \prime},
$$

где

$$
E^{\prime \prime} \subseteq\left\{\left\{\left(i_{1}, \ldots, i_{d-1}, i_{d}, k\right),\left(i_{1}, \ldots, i_{d-1}, i_{d}+1, l\right)\right\}: i_{1}, \ldots, i_{d} \in \mathbb{Z}, k, l \in\{1,2\}\right\},
$$

причем (в силу $c=0$ ) для произвольных $i_{1}, \ldots, i_{d} \in \mathbb{Z}$ множество $E_{i_{1}, \ldots, i_{d}}:=$ $\left\{(k, l): k, l \in\{1,2\},\left\{\left(i_{1}, \ldots, i_{d-1}, i_{d}, k\right),\left(i_{1}, \ldots, i_{d-1}, i_{d}+1, k\right)\right\} \in E^{\prime \prime}\right\}$ совпадает с одним из множеств: $\{(1,1),(2,2)\},\{(1,2),(2,1)\}$. (Вообще говоря, множество $E_{i_{1}, \ldots, i_{d}}$ зависит от выбора $i_{1}, \ldots, i_{d} \in \mathbb{Z}$.) Не теряя общности (меняя местами, 
в случае необходимости, вершины внутри блоков $\sigma$, принадлежащих множествам $\varphi_{i}\left(V\left(\Gamma^{\prime}\right)\right)$ для подходящих $i \in \mathbb{Z}$ ), можно считать, что (наряду с (а) и (b)) справедливо следующее утверждение:

(c) $E_{0, \ldots, 0, i}=\{(1,1),(2,2)\}$ для всех $i \in \mathbb{Z}$.

Теперь для завершения доказательства утверждения 2) теоремы покажем индукцией по $n=\left|i_{1}\right|+\cdots+\left|i_{d-1}\right|$, где $i_{1}, \ldots, i_{d-1} \in \mathbb{Z}$, что для каждого $i \in \mathbb{Z}$ множество $E_{i_{1}, \ldots, i_{d-1}, i}$ однозначно определяется множеством $\mathcal{P}$.

При $n=0$ справедливость утверждения следует из (с). Предположим, что утверждение справедливо при всех $n \leqslant n_{0}$, где $n_{0}-$ некоторое целое неотрицательное число, и пусть $i_{1}, \ldots, i_{d-1} \in \mathbb{Z}$ такие, что $\left|i_{1}\right|+\cdots+\left|i_{d-1}\right|=n_{0}+1$. Тогда для некоторого $1 \leqslant j \leqslant d-1$ имеем $i_{j} \neq 0$. Положим $i_{k}^{\prime}=i_{k}$ при $1 \leqslant k \leqslant d-1$ и $k \neq j$, и пусть $i_{j}^{\prime}:=i_{j}-\varepsilon$, где $\varepsilon=1$ при $i_{j}>0$ и $\varepsilon=-1$ при $i_{j}<0$. Тогда $\left|i_{1}^{\prime}\right|+\cdots+\left|i_{d-1}^{\prime}\right|=n_{0}$, и по предположению индукции для каждого $i \in \mathbb{Z}$ множество $E_{i_{1}^{\prime}, \ldots, i_{d-1}^{\prime}, i}$ однозначно определяется множеством $\mathcal{P}$.

Пусть $x$ - произвольная вершина графа $\Gamma$ из $\left\{\left(i_{1}, \ldots, i_{d-1}, i, 1\right),\left(i_{1}, \ldots, i_{d-1}\right.\right.$, $i, 2)\}$ и $y-$ произвольная вершина графа $\Gamma$ из $\left\{\left(i_{1}, \ldots, i_{d-1}, i+1,1\right),\left(i_{1}, \ldots, i_{d-1}\right.\right.$, $i+1,2)\}$. По $x$ и $y$ однозначно определяются вершина $x^{\prime} \in\left\{\left(i_{1}^{\prime}, \ldots, i_{d-1}^{\prime}, i, 1\right)\right.$, $\left.\left(i_{1}^{\prime}, \ldots, i_{d-1}^{\prime}, i, 2\right)\right\}$ и вершина $y^{\prime} \in\left\{\left(i_{1}^{\prime}, \ldots, i_{d-1}^{\prime}, i+1,1\right),\left(i_{1}^{\prime}, \ldots, i_{d-1}^{\prime}, i+1,2\right)\right\}$ такие, что $\left\{x, x^{\prime}\right\},\left\{y, y^{\prime}\right\} \in E(\Gamma)$. (Вершины $x^{\prime}, y^{\prime}$ определяются условиями $\left\{\varphi_{i}^{-1}\left(x^{\prime}\right), \varphi_{i+1}^{-1}\left(y^{\prime}\right)\right\} \in\left\{\left(i_{1}^{\prime}, \ldots, i_{d-1}^{\prime}, 1\right),\left(i_{1}^{\prime}, \ldots, i_{d-1}^{\prime}, 2\right)\right\},\left\{\varphi_{i}^{-1}(x), \varphi_{i}^{-1}\left(x^{\prime}\right)\right\} \in$ $E\left(\Gamma^{\prime}\right),\left\{\varphi_{i+1}^{-1}(y), \varphi_{i+1}^{-1}\left(y^{\prime}\right)\right\} \in E\left(\Gamma^{\prime}\right)$.) Если $\{j, d\} \in \mathcal{P}$, то $\{x, y\} \in E_{\left(i_{1}, \ldots, i_{d-1}, i\right)}$ тогда и только тогда, когда $\left\{x^{\prime}, y^{\prime}\right\} \in E_{\left(i_{1}^{\prime}, \ldots, i_{d-1}^{\prime}, i\right)}$. Если $\{j, d\} \notin \mathcal{P}$, то $\{x, y\} \in E_{\left(i_{1}, \ldots, i_{d-1}, i\right)}$ тогда и только тогда, когда $\left\{x^{\prime}, y^{\prime}\right\} \notin E_{\left(i_{1}^{\prime}, \ldots, i_{d-1}^{\prime}, i\right)}$. Таким образом, $E_{\left(i_{1}, \ldots, i_{d-1}, i\right)}$ однозначно определяется по $E_{\left(i_{1}^{\prime}, \ldots, i_{d-1}^{\prime}, i\right)}$ и $\mathcal{P}$, что завершает доказательство утверждения 2) теоремы.

Докажем утверждение 3$)$. Пусть $\Gamma-\operatorname{Aut}_{0}\left(\Lambda^{d}\right)$-симметрическое 2-расширение решетки $\Lambda^{d}$, реализуемое $G, \sigma, \varphi$ и реализующее относительно изоморфизма $\varphi$ набор $(c, \mathcal{P})$, и $\Gamma^{*}-\operatorname{Aut}_{0}\left(\Lambda^{d}\right)$-симметрическое 2 -расширение решетки $\Lambda^{d}$, реализуемое $G^{*}, \sigma^{*}, \varphi^{*}$ и реализующее относительно изоморфизма $\varphi^{*}$ набор $\left(c^{*}, \mathcal{P}^{*}\right)$.

Предположим, что имеется изоморфизм $\chi: \Gamma \rightarrow \Gamma^{*}$. Покажем, что в этом случае $c^{*}=c$ и существует подстановка на $\{c+1, \ldots, d\}$, переводящая $\mathcal{P}$ в $\mathcal{P}^{*}$. Согласно предложению 4.2 изоморфизм $\chi$ графа $\Gamma$ на граф $\Gamma^{*}$ индуцирует изоморфизм $\bar{\chi}$ графа $\Gamma / \sigma$ на граф $\Gamma^{*} / \sigma^{*}$. Положим $\omega=\varphi^{*} \bar{\chi} \varphi^{-1} \in \operatorname{Aut}\left(\Lambda^{d}\right)$. Для каждого $1 \leqslant i \leqslant d$ обозначим через $\Lambda_{\{i\}}^{d}$ множество $\left\{x \in V\left(\Lambda^{d}\right): \operatorname{Pr}_{j}(x)=0\right.$, $1 \leqslant j \leqslant d, j \neq i\}$. Автоморфизм $\omega$ решетки $\Lambda^{d}$ задает на множестве $\{1, \ldots, d\}$ подстановку $\pi_{\omega}$ такую, что $\omega\left(\Lambda_{\{i\}}^{d}\right)=\Lambda_{\left\{\pi_{\omega}(i)\right\}}^{d}$ для всех $i \in\{1, \ldots, d\}$. Ясно, что для произвольного $1 \leqslant i \leqslant d$ в $Г$ относительно $\varphi$ направление $\{i\}$ реализует тип $\Gamma_{2}^{1,2}$ тогда и только тогда, когда в $\Gamma^{*}$ относительно $\varphi^{*}$ направление $\left\{\pi_{\omega}(i)\right\}$ реализует тип $\Gamma_{2}^{1,2}$. Ясно также, что для произвольных $1 \leqslant i<j \leqslant d$ в $Г$ относительно $\varphi$ пара направлений $\{i, j\}$ реализует тип $\Gamma_{1}^{2,2}$ тогда и только тогда, когда в $\Gamma^{*}$ относительно $\varphi^{*}$ пара направлений $\left\{\pi_{\omega}(i), \pi_{\omega}(j)\right\}$ реализует тип $\Gamma_{1}^{2,2}$. Поскольку $\{1, \ldots, c\}=\{i: 1 \leqslant i \leqslant d$ и в Г относительно $\varphi$ направление $\{i\}$ реализует тип $\left.\Gamma_{2}^{1,2}\right\}$ и $\left\{1, \ldots, c^{*}\right\}=\left\{i: 1 \leqslant i \leqslant d\right.$ и в $\Gamma^{*}$ относительно $\varphi^{*}$ направление $\{i\}$ реализует тип $\left.\Gamma_{2}^{1,2}\right\}$, то $c^{*}=c$ и $\{1, \ldots, c\}-\pi_{\omega}$-инвариантное 
множество. Кроме того, поскольку $\mathcal{P}=\{\{i, j\}: 1 \leqslant i<j \leqslant d$ и в Г относительно $\varphi$ пара направлений $\{i, j\}$ реализует тип $\left.\Gamma_{1}^{2,2}\right\}$ и $\mathcal{P}^{*}=\{\{i, j\}: 1 \leqslant i<j \leqslant d$ и в $\Gamma^{*}$ относительно $\varphi^{*}$ пара направлений $\{i, j\}$ реализует тип $\left.\Gamma_{1}^{2,2}\right\}$, то $\pi_{\omega}$ переводит $\mathcal{P}$ в $\mathcal{P}^{*}$.

Покажем, что обратное утверждение также справедливо, т. е. что из $c=c^{*}$ и существования подстановки на $\{c+1, \ldots, d\}$, переводящей $\mathcal{P}$ в $\mathcal{P}^{*}$, следует изоморфизм графов $Г$ и $\Gamma^{*}$. Пусть $\pi$ - подстановка на $\{c+1, \ldots, d\}$, переводящая $\mathcal{P}$ в $\mathcal{P}^{*}$. Следующим образом определим подстановку $\widetilde{\pi}$ на $\{1, \ldots, d\}: \widetilde{\pi}(i)=i$ при $i \leqslant c$ и $\tilde{\pi}(i)=\pi(i)$ при $i>c$. Найдется такой автоморфизм $\omega$ решетки $\Lambda^{d}$, что $\tilde{\pi}$ совпадает с подстановкой $\pi_{\omega}$ на $\{1, \ldots, d\}$, определяемой по правилу: $\omega\left(\Lambda_{\{i\}}^{d}\right)=\Lambda_{\left\{\pi_{\omega}(i)\right\}}^{d}$ для всех $i \in\{1, \ldots, d\}$, где $\Lambda_{\{i\}}^{d}=\left\{x \in V\left(\Lambda^{d}\right): \operatorname{Pr}_{j}(x)=0\right.$, $1 \leqslant j \leqslant d, j \neq i\}$ для каждого $1 \leqslant i \leqslant d$. Для так определенного автоморфизма $\omega$ граф $\Gamma^{*}$ реализует набор $(c, \mathcal{P})$ относительно изоморфизма $\omega^{-1} \varphi^{*}: \Gamma^{*} / \sigma^{*} \rightarrow \Lambda^{d}$. В силу утверждения 2) теоремы отсюда следует, что граф $Г$ изоморфен графу $\Gamma^{*}$.

Утверждение 4) очевидно. Теорема доказана.

ЗАмЕЧАНИЕ 5.4. Согласно замечанию 5.1 для любого целого положительного числа $d$ имеется единственное (с точностью до изоморфизма) несвязное обобщенное $\operatorname{Aut}_{0}\left(\Lambda^{d}\right)$-симметрическое 2-расширение решетки $\Lambda^{d}$, которое является дизъюнктным объединением двух графов, изоморфных $\Lambda^{d}$. Это несвязное обобщенное $\operatorname{Aut}_{0}\left(\Lambda^{d}\right)$-симметрическое 2-расширение решетки $\Lambda^{d}$ изоморфно $\nu(\Gamma)$, где $\Gamma$ - насыщенное $\operatorname{Aut}_{0}\left(\Lambda^{d}\right)$-симметрическое 2-расширение решетки $\Lambda^{d}$, реализующее набор $(0,\{\{i, j\}: 1 \leqslant i<j \leqslant d\})$.

ЗАмЕЧАНИЕ 5.5. Зафиксируем произвольное целое положительное число $d$. Пусть $\Gamma$ - насыщенное $\operatorname{Aut}_{0}\left(\Lambda^{d}\right)$-симметрическое 2-расширение решетки $\Lambda^{d}$, реализующее относительно изоморфизма $\varphi$ (графа $\Gamma / \sigma$, где $\sigma$ - соответствующая $Г$ система блоков, на решетку $\Lambda^{d}$ ) набор $(c, \mathcal{P})$ (по утверждению 1 ) теоремы 5.1 для каждого Г такие $\varphi$ и $(c, \mathcal{P})$ существуют). По Г и $\varphi$ следующим образом определим граф $[\Gamma]_{\varphi}: V\left([\Gamma]_{\varphi}\right)=\{c+1, \ldots, d\}, E\left([\Gamma]_{\varphi}\right)=\mathcal{P}$.

Согласно утверждению 2) теоремы 5.1 каждый конечный граф с не более чем $d$ вершинами изоморфен графу $[\Gamma]_{\varphi}$ для подходящих $\Gamma$ и $\varphi$. Далее, если $\Gamma$, помимо реализуемости относительно изоморфизма $\varphi$ набора $(c, \mathcal{P})$, реализует относительно изоморфизма $\varphi^{\prime}$ набор $\left(c^{\prime}, \mathcal{P}^{\prime}\right)$, то согласно утверждению 3$)$ теоремы 5.1 графы $[\Gamma]_{\varphi}$ и $[\Gamma]_{\varphi^{\prime}}$ изоморфны. Более того, согласно утверждению 3) теоремы 5.1 если для каждого $k \in\{1,2\}$ граф $\Gamma_{k}$ является насыщенным $\operatorname{Aut}_{0}\left(\Lambda^{d}\right)$-симметрическим 2-расширением решетки $\Lambda^{d}$, реализующим относительно изоморфизма $\varphi_{k}$ набор $\left(c_{k}, \mathcal{P}_{k}\right), 0 \leqslant c_{k} \leqslant d, \mathcal{P}_{k} \subseteq\left\{\{i, j\}: c_{k}<i<\right.$ $j \leqslant d\}$, то графы $\Gamma_{1}$ и $\Gamma_{2}$ изоморфны тогда и только тогда, когда изоморфны графы $\left[\Gamma_{1}\right]_{\varphi_{1}}$ и $\left[\Gamma_{2}\right]_{\varphi_{2}}$. Таким образом, существует биективное соответствие между множеством изоморфных типов насыщенных $\operatorname{Aut}_{0}\left(\Lambda^{d}\right)$-симметрических 2 -расширений решетки $\Lambda^{d}$ и множеством изоморфных типов конечных графов с не более чем $d$ вершинами (включая граф с пустым множеством вершин).

Теорема 5.1 позволяет получить формулу для числа $N_{d, 2}$ (попарно неизоморфных) $\operatorname{Aut}_{0}\left(\Lambda^{d}\right)$-симметрических 2-расширений решетки $\Lambda^{d}$, где $d$ - произ- 
вольное целое положительное число. Чтобы сформулировать соответствующее утверждение (см. следствие 5.1 далее), для каждого целого положительного числа $k$ обозначим через $N_{k}$ число (попарно неизоморфных) графов с $k$ вершинами. Тогда (см., например, [13, теорема 15.5])

$$
N_{k}=\sum_{\substack{\left(j_{1}, j_{2}, \ldots, j_{k}\right) \in \mathbb{Z}^{k} \\ j_{1}, j_{2}, \ldots, j_{k} \geqslant 0 \\ j_{1}+2 j_{2}+\cdots+k j_{k}=k}} \frac{2^{m_{\left(j_{1}, j_{2}, \ldots, j_{k}\right)}}}{\prod_{i=1}^{k} j_{i} ! i^{j_{i}}}
$$

где

$$
m_{\left(j_{1}, j_{2}, \ldots, j_{k}\right)}=\sum_{i=1}^{\left[\frac{k}{2}\right]} i\left(j_{2 i}+\frac{j_{i}\left(j_{i}-1\right)}{2}\right)+\sum_{i=1}^{\left[\frac{k-1}{2}\right]} i j_{2 i+1}+\sum_{1 \leqslant l<m \leqslant k-1} \text { НОД }(l, m) j_{l} j_{m} .
$$

СлЕДСТвИЕ 5.1. Для произвольного иелого положительного числа d имеем

$$
N_{d, 2}=2\left(N_{1}+\cdots+N_{d}\right)+1 \text {. }
$$

ДокАЗАтельство. Согласно замечанию 5.5 число (попарно неизоморфных) насыщенных $\operatorname{Aut}_{0}\left(\Lambda^{d}\right)$-симметрических 2-расширений решетки $\Lambda^{d}$ равно $1+N_{1}+\cdots+N_{d}$. В силу утверждения 4) теоремы 5.1 и замечания 5.4 отсюда следует, что число всех (попарно неизоморфных) $\operatorname{Aut}_{0}\left(\Lambda^{d}\right)$-симметрических 2 -расширений решетки $\Lambda^{d}$ равно $1+2\left(N_{1}+\cdots+N_{d}\right)$ (а число всех обобщенных $\operatorname{Aut}_{0}\left(\Lambda^{d}\right)$-симметрических 2-расширений решетки $\Lambda^{d}$ равно $\left.2\left(1+N_{1}+\cdots+N_{d}\right)\right)$.

При $d \leqslant 10$ следствие 5.1 дает для $N_{d, 2}$ приводимые ниже значения:

$$
\begin{array}{ll}
N_{1,2}=3, & N_{6,2}=417, \\
N_{2,2}=7, & N_{7,2}=2505, \\
N_{3,2}=15, & N_{8,2}=27197 \\
N_{4,2}=37, & N_{9,2}=576533 \\
N_{5,2}=105, & N_{10,2}=24586869 .
\end{array}
$$

Из следствия 5.1 и [14, формула (9.1.2)] вытекает справедливость следующего утверждения.

СлЕДСТВиЕ 5.2. При $d \rightarrow \infty$ имеем

$$
N_{d, 2}=\frac{2 \cdot 2^{\frac{d(d-1)}{2}}}{d !}\left(1+\frac{d^{2}-d}{2^{d-1}}\right)+O\left(\frac{2^{\frac{d(d-3)}{2}}}{(d-1) !}\right) .
$$

ЗАмЕчАниЕ 5.6. Еще одним следствием теоремы 5.1 является выполнение для произвольного $\operatorname{Aut}_{0}\left(\Lambda^{d}\right)$-симметрического 2-расширения решетки $\Lambda^{d}$ условия $\left[n_{1}, \ldots, n_{d}\right]$-периодичности, где $n_{1}, \ldots, n_{d} \leqslant 2$. Точнее, справедливо следующее утверждение. Пусть $\Gamma-\operatorname{Aut}_{0}\left(\Lambda^{d}\right)$-симметрическое 2-расширение решетки $\Lambda^{d}, d \geqslant 1$, реализующее набор $(c, \mathcal{P})$ относительно некоторого изоморфизма $\varphi$ графа $\Gamma / \sigma$, где $\sigma$ - соответствующая $\Gamma$ система блоков, на решетку $\Lambda^{d}$. 
Тогда $(\Gamma, \sigma, \varphi)$ удовлетворяет условию $\left[n_{1}, \ldots, n_{d}\right]$-периодичности, где $n_{1}, \ldots, n_{d}$ выбираются следующим образом: фиксируется произвольная максимальная клика $K$ графа $[\Gamma]_{\varphi}$ (см. замечание 5.5$)$, после чего $n_{i}$ полагается равным 1 для всех $1 \leqslant i \leqslant c$ и всех $i$, являющихся вершинами клики $K$, и полагается равным 2 для всех $c<i \leqslant d$, не являющихся вершинами клики $K$.

ЗАмЕчАНИЕ 5.7. Основные результаты настоящего параграфа ранее были анонсированы в [15].

\section{Список литературы}

1. V.I. Trofimov, "Symmetrical extensions of graphs and some other topics in graph theory related with group theory", Тр. ИММ УрО РАН, 17:4 (2011), 316-320; Proc. Steklov Inst. Math. (Suppl.), 279, suppl. 1 (2012), 107-112.

2. W. Imrich, S. Klavžar, Product graphs. Structure and recognition, Wiley-Intersci. Ser. Discrete Math. Optim., Wiley-Interscience, New York, 2000, xvi+358 pp.

3. В.И. Трофимов, "Графы с полиномиальным ростом", Матем. сб., 123(165):3 (1984), 407-421; англ. пер.: V. I. Trofimov, "Graphs with polynomial growth", Math. USSR-Sb., 51:2 (1985), 405-417.

4. W. Woess, "Topological groups and recurrence of quasi tranitive graphs", Rend. Sem. Mat. Fis. Milano, 64:1 (1996), 185-213.

5. В.И. Трофимов, “Ограниченные автоморфизмы графов и одна характеризация решеток", Изв. АН СССР. Сер. матем., 47:2 (1983), 407-420; англ. пер.: V.I. Trofimov, "Automorphisms of graphs and a characterization of lattices", Math. USSR-Izv., 22:2 (1984), 379-391.

6. М. Грин, Дж. Шварц, Э. Виттен, Теория суперструн, т. 1, 2, Мир, М., 1990, 520 с., 656 с.; пер. с англ.: М.В. Green, J. H. Schwarz, E. Witten, Superstring theory, v. 1, 2, Cambridge Monogr. Math. Phys., Cambridge Univ. Press, Cambridge, 1987, x+469 pp., xii+596 pp.

7. Е. А. Неганова, В. И. Трофимов, "О симметрических $q$-расширениях 2-мерной решетки", Тр. ИММ УрО РАН, 16:3 (2010), 199-209; англ. пер.: Е. A. Neganova, V. I. Trofimov, "On symmetrical $q$-extensions of the 2-dimensional grid", Proc. Steklov Inst. Math. (Suppl.), 272, suppl. 1 (2011), 127-137.

8. Е. А. Неганова, В. И. Трофимов, "О симметрических 4-расширениях 2-мерной решетки”, Тр. ИММ УрО РАН, 17:3 (2011), 242-257.

9. Е.А. Неганова, "Aut $\left(\Lambda^{2}\right)$-симметрические 4-расширения 2-мерной решетки $\Lambda^{2}$ ", Тр. ИММ УрО РАН, 17:4 (2011), 222-243; англ. пер.: Е. A. Neganova, "Aut ${ }_{0}\left(\Lambda^{2}\right)$ symmetrical 4-extensions of the 2-dimensional grid $\Lambda^{2 "}$, Proc. Steklov Inst. Math. (Suppl.), 279, suppl. 1 (2012), 84-106.

10. N. Seifter, V. I. Trofimov, "Automorphism groups of graphs with quadratic growth", J. Combin. Theory Ser. B, 71:2 (1997), 205-210.

11. G. Baumslag, R. Strebel, M. W. Thomson, "On the multiplicator of $F / \gamma_{c} R$ ", J. Pure Appl. Algebra, 16:2 (1980), 121-132.

12. А. Ю. Ольшанский, Геометрия определяющих соотношений в группах, Современная алгебра, Наука, М., 1989, 448 с.; англ. пер.: А. Yu. Ol'shanskiı̌, Geometry of defining relations in groups, Math. Appl. (Soviet Ser.), 70, Kluwer Acad. Publ., Dordrecht, 1991, xxvi+505 pp.

13. Ф. Харари, Теория графов, Мир, М., 1973, 300 с.; пер. с англ.: F. Harary, Graph theory, Addison-Wesley Publishing Co., Reading, Mass.-Menlo Park, Calif.-London, 1969, ix +274 pp. 
14. Ф. Харари, Э. Палмер, Перечисление графов, Мир, М., 1977, 324 с.; пер. с англ.: F. Harary, E. M. Palmer, Graphical enumeration, Academic Press, New York-London, 1973, xiv+271 pp.

15. Е. А. Неганова, В. И. Трофимов, " $\mathrm{Aut}_{0}\left(\Lambda^{d}\right)$-симметрические 2-расширения решеток $\Lambda^{d}$ ", Тез. докладов 41-й Всероссийской молодежной конферениии "Проблемы теоретической и прикладной математики", ИММ УрО РАН, Екатеринбург, 2010, 64-70.

ЕЛЕНА АЛЕКСАНДРОвНА НЕГАНОВА

Поступило в редакцию

(Elena A. Neganova)

01.10 .2012

Институт математики и механики УрО РАН,

г. Екатеринбург

E-mail: nega-le@yandex.ru

ВЛАДИМИР ИвАНОВИч ТРОФИМОВ

(Vladimir I. Trofimov)

Институт математики и механики УрО РАН;

Уральский федеральный университет,

г. Екатеринбург

E-mail: trofimov@imm.uran.ru 